\title{
Differentiation of Axon-Related Schwann Cells in vitro: II. Control of Myelin Formation by Basal Lamina
}

\author{
Charles F. Eldridge, ${ }^{a}$ Mary Bartlett Bunge, and Richard P. Bunge \\ Department of Anatomy and Neurobiology, Washington University School of Medicine, St. Louis, Missouri 63110
}

Several recent observations suggest that Schwann cell (SC) differentiation, including myelin formation, is dependent upon the development of basal lamina which characteristically surrounds each axon-SC unit in peripheral nerve. This dependence can be tested in a neuron-SC culture system developed in our laboratory in which SC differentiation, including basal lamina formation and myelination, is faithfully reproduced. The use of serum-free, defined medium (DM) with this culture system allows axon-driven SC proliferation but not basal lamina formation or myelination. We previously demonstrated that ascorbic acid, in the presence of a nondialyzable serum factor(s), stimulates basal lamina assembly and myelin formation with similar dose-response relationships (Eldridge et al., 1987). We hypothesized that ascorbic acid acts to promote SC myelination indirectly, by enabling the assembly of basal lamina. We now provide support for this hypothesis by demonstrating the following. (1) Pepsin-resistant triple-helical collagen molecules were produced only by SCs grown in the presence of ascorbic acid, suggesting that triple-helical type IV collagen may mediate the effect of ascorbic acid on basal lamina formation. (2) The formation of myelin by oligodendrocytes, which myelinate axons in the CNS without the concomitant deposition of basal lamina, was little affected by ascorbic acid, suggesting that the biosynthesis and assembly of myelin per se does not require ascorbic acid. (3) The provision of exogenous basal lamina matrix to SCs grown with neurons in DM without ascorbic acid promoted control levels of myelination (and basal lamina formation); the provision of exogenous fibrillar collagen matrix did not. (4) Purified laminin promoted control levels of myelination in the absence of ascorbic acid, but purified type IV collagen and heparan sulfate proteoglycan (HSPG) did not. Laminin caused SCs to assemble basal lamina-like structures that contained not only laminin but also

\footnotetext{
Received Apr. 13, 1988; revised June 21, 1988; accepted June 29, 1988.

We wish to thank Drs. E. Crouch, P. Wood, and J. Sanes for advice and comments on the manuscript, Dr. Crouch for purified laminin, Dr. Hassell for purified HSPG, Drs. J. Brockes, Crouch, A. Charonis, C. Cornbrooks, and R. Timpl for antibodies, Dr. E. Johnson for purified NGF, Margaret Bates for electron microscopic preparations and photography, Joe Hayes for help with photography, Susan Mantia for word processing, and Laura Tynan and Artree James for technical assistance. This work was done in partial fulfillment of the requirements for the Ph.D degree. Supported by NIH Grant NS09923 and also Training Grant NS07071 to C.F.E.

Correspondence should be addressed to Mary Bartlett Bunge, Department of Anatomy and Neurobiology, Washington University School of Medicine, 660 South Euclid Avenue, St. Louis, MO 63110.

a Present address: Department of Biochemistry, J405 Health Sciences Bldg., SJ70, University of Washington, Seattle, WA 98195.

Copyright (C) 1989 Society for Neuroscience $0270-6474 / 89 / 020625-14 \$ 02.00 / 0$
}

HSPG and non-triple-helical type IV collagen. Thus, several types of experiments demonstrate that SC myelin formation can be controlled by regulating the ability of the SC to assemble basal lamina, illustrating that acquisition of basal lamina is a crucial prefatory step for further SC differentiation.

The differentiation of certain cells of neural crest origin into SCs of the peripheral nerve trunk involves a number of stages, including basal lamina formation, proliferation, and eventually ensheathment or myelination of axons (reviewed in Webster and Favilla, 1984). Although these stages overlap temporally during development in vivo, they can be experimentally separated, both in vivo and in vitro (reviewed in Bunge and Bunge, 1984), and thus can be studied relatively independently from one another. The neuron, via its axon, has been shown to exert control over several stages of Schwann cell (SC) differentiation; these include the expression of basal lamina components (Carey et al., 1983; Dean et al., 1986) and formation of basal lamina (Bunge et al., 1982), SC mitosis (Wood and Bunge, 1975; McCarthy and Partlow, 1976; Salzer and Bunge, 1980; Salzer et al., 1980a, b; DeVries et al., 1982; Ratner et al., 1987), and the expression and processing of myelin proteins and lipids (Mirsky et al., 1980; Brockes et al., 1981; Politis et al., 1982; Poduslo et al., 1985; Brunden and Poduslo, 1987).

Tissue culture studies have shown that, in addition to the axon, the basal lamina may also play important roles in SC differentiation. Two glycoproteins related to basal lamina, laminin and fibronectin, have been shown to stimulate $\mathrm{SC}$ division by unknown mechanisms (Baron-Van Evercooren et al., 1982; McGarvey et al., 1984; Eldridge and Bunge, 1987). Work from our laboratory has demonstrated that there exists a close correlation between the ability of the SC to form a basal lamina and to myelinate axons. Moya et al. (1980) initially reported that SCs cultured with neurons in serum-free defined medium (DM) are able to proliferate in response to axonal contact but do not form either basal lamina or myelin. Providing such SCneuron cultures with standard myelinating medium, containing serum and chick embryo extract, results in the assembly of basal laminae and the establishment of normal SC-axon relationships, including myelination. We have recently presented evidence (Eldridge et al., 1987) that the components of standard myelinating medium essential for the promotion of both basal lamina assembly and myelination are ascorbic acid (the critical component of embryo extract) and a nondialyzable serum component(s). The serum component(s) alone has little detectable effect but is essential for allowing ascorbic acid to manifest its ability to promote these aspects of SC differentiation; ascorbic 
acid in the absence of serum promotes only the irregular deposition of basal lamina components and the formation of small quantities of myelin.

These results suggested that the SC requires basal lamina in order to form myelin and that ascorbic acid enables the SC to assemble basal lamina rather than influencing myelination directly. The results of the present study support this concept of the mechanism by which ascorbic acid influences peripheral myelination.

\section{Materials and Methods}

Cell culture. Dissociated cell cultures containing purified populations of sensory neurons and SCs were prepared from embryonic day (E) 15 Sprague-Dawley rat dorsal root ganglia as previously described (Bunge et al., 1983; Eldridge et al., 1987). Briefly, cultures were cycled on and off $10^{-5} \mathrm{M}$ fluorodeoxyuridine in serum-containing medium for 2 weeks, grown in DM for 1 week, seeded with $1-2 \times 10^{4}$ purified $S C s$ per culture, and maintained in DM for 4 weeks to allow the generation of a full SC population. DM contained insulin, transferrin, progesterone, putrescine, selenium, and 2.5S NGF in a 1:1 mixture of Dulbecco's modified Eagle's medium and Ham's F-12 (GIBCO, Grand Island, NY); the total protein concentration was $15.1 \mu \mathrm{g} / \mathrm{ml}$. Fully repopulated SC-neuron cultures were maintained in DM or various supplemented DMs for 2 weeks, after which time they were metabolically labeled or processed for light or electron microscopy (see below).

Cultures for study of oligodendrocyte (ODC) myelination were prepared as described for SC-neuron cultures, except seeding used dissociated E19 rat lumbar spinal cord (Wood and Williams, 1984) at $50 \times$ $10^{3}$ cells per culture. After seeding, cultures were maintained in DM for 2 weeks and then in DM or various supplemented DMs for 4 weeks. To detect myelin-forming SC colonies that form in such cultures (Wood and Williams, 1984), immunocytochemistry (see below) was performed on fixed and permeabilized cultures (Bologna-Sandru et al., 1981) using antiserum to $P_{o}$ (a gift from Dr. J. Brockes; Brockes et al., 1980). The location of all $\mathrm{P}_{0}^{+}$, myelin-forming SC colonies was mapped onto graph paper by scanning at $250 \times$. Cultures were then unmounted, processed for Sudan black staining, and remounted, maintaining orientation. Fields arrayed at $1 \mathrm{~mm}$ intervals on a grid covering most of the culture were evaluated at $640 \times$ for the presence of myelin; the density of CNS myelin made quantitation of the number of myelin segments impossible. Fields that had been previously determined to contain $\mathrm{P}_{\mathrm{o}}+$ myelin were scored as negative for CNS myelin.

$D M$ supplementation. Details have been provided previously (Eldridge et al., 1987). Briefly, serum used for supplementation was human placental cord serum (HPS; prepared from blood obtained locally) or fetal bovine serum (FBS; Hyclone Labs, Logan, UT). In most cases (as indicated), the serum was dialyzed against DM salt base before use in supplementing DM; serum was dialyzed against leucine-free Eagle's minimal essential medium for use in metabolic labeling experiments. L-Ascorbic acid (Sigma, St. Louis) was used at $280 \mu \mathrm{M}(50 \mu \mathrm{g} / \mathrm{ml})$. Chick embryo extract (Paul, 1970) was used at 5 or $10 \%$ (vol/vol). Myelinating medium (MM) contained HPS plus embryo extract (Moya et al., 1980) or dialyzed FBS plus ascorbic acid (Eldridge et al., 1987), as indicated.

Gel overlays. In some experiments, fully populated SC-ncuron cultures grown in DM were overlaid with either a fibrous collagen gel (FCG) or a basal lamina gel (BLG) immediately before initiating a DM supplementation experiment. FCGs were prepared by the method of Elsdale and Bard (1972); type I collagen-rich rat-tail collagen (prepared in the laboratory by the method of Bornstein, 1958) in $0.1 \%$ acetic acid was mixed on ice with $1 / 10$ vol of a $2: 1$ mixture of $10 \times$ Hank's balanced salt solution: $0.34 \mathrm{~N} \mathrm{NaOH}$. The culture medium was drained from the cultures, $0.25 \mathrm{ml}$ of the neutralized collagen solution was added, and the cultures were maintained at $35^{\circ} \mathrm{C}$ for $20-30 \mathrm{~min}$ in order for a gel to form. BLGs were prepared by pipetting $0.25 \mathrm{ml}$ of ice-cold basal lamina matrix [Matrigel, a whole extract of Englebreth-Holm-Swarm (EHS) sarcoma extracellular matrix, (Kleinman et al., 1986) Collaborative Research, Lexington, $\mathrm{MA}$ ] onto drained cultures and then maintaining them at $35^{\circ} \mathrm{C}$ for $20-30 \mathrm{~min}$. $\Lambda$ fter gel formation, cultures were given $\mathrm{DM}$, maintained at $35^{\circ} \mathrm{C}$ for $90 \mathrm{~min}$, and then refed $\mathrm{DM}$ or supplemented DM. In some experiments, basal lamina matrix was diluted into ice-cold DM and used as culture medium.

Purified basal lamina components. A sterile solution of laminin in 50 $\mathrm{mm}$ Tris/150 $\mathrm{mm} \mathrm{NaCl}$, pH 7.4, purified from the EHS sarcoma was obtained from Collaborative Research. EHS sarcoma laminin was also obtained from Bethesda Research Laboratories (Gaithersburg, MD) and as a gift from Dr. E. Crouch (Dept. Pathology, Jewish Hosp., St. Louis, MO); when laminin from these sources was used, all media were filtersterilized. Type IV collagen in $0.5 \mathrm{~m}$ acetic acid purified from the EHS sarcoma was obtained from Collaborative Research and from Bethesda Research Laboratories; it was dialyzed aseptically into DM salt base before use. Large, low-density HSPG purified from the EHS sarcoma (Hassell et al., 1985) was generously supplied by Dr. John Hassell (NIDR, $\mathrm{NIH}$, Bethesda, MD); the lyophilized proteoglycan was dissolved in 4 $\mathrm{M}$ guanidine- $\mathrm{HCl} / 50 \mathrm{~mm}$ phosphate, $\mathrm{pH} 7.4$, and then dialyzed aseptically into PBS.

Metabolic labeling of cultures. Triplicate SC-neuron cultures were labeled for $24 \mathrm{hr}$ at the end of the 2 week supplementation period. Cultures grown in DM were labeled in medium A [leucine-free Eagle's MEM containing 2.5S NGF, transferrin, insulin, putrescine, progesterone, and selenium at the same concentrations as in DM, and with 5.2 $\mu \mathrm{g} / \mathrm{ml} \mathrm{L}$-leucine ( $10 \%$ of the normal concentration) and $200 \mu \mathrm{Ci} / \mathrm{ml}$ L-3,4,5-3 $\mathrm{H}$-leucine (New England Nuclear, Boston]. Cultures grown in DM supplemented with ascorbic acid were labeled in medium A plus $50 \mu \mathrm{g} / \mathrm{ml}$ ascorbic acid. Cultures grown in standard myelinating medium (Moya et al., 1980) or DM supplemented with HPS plus embryo extract were labeled in medium A plus $5 \%$ dialyzed HPS plus $50 \mu \mathrm{g} / \mathrm{ml}$ ascorbic acid. Cultures grown in DM supplemented with dialyzed FBS with or without ascorbic acid were labeled in medium A plus 5\% dialyzed FBS with or without $50 \mu \mathrm{g} / \mathrm{ml}$ ascorbic acid, respectively. In one experiment, cultures in DM were labeled with ${ }^{3} \mathrm{H}$-leucine either in leucine-free $\mathrm{Ea}$ gle's MEM plus NGF as described by Carey and Bunge (1981), or simply in DM. In 2 experiments, cultures were labeled with $200 \mu \mathrm{Ci} / \mathrm{ml} \mathrm{L}^{-35} \mathrm{~S}-$ methionine in the media in which they were being grown. Labeling media were filter-sterilized; cultures were labeled in $0.4-0.65 \mathrm{ml}$, and total incorporation of labeled precursor was $<10 \%$ of available precursor.

At the end of the labeling period, media and cell layers were separated and protease inhibitors (Eldridge et al., 1986) added, and all medium samples were made to $4 \%$ serum (by adding dialyzed serum or medium A to serum-free or serum-containing samples, respectively). Incorporation of ${ }^{3} \mathrm{H}$-leucine into protein in medium and sonicated cell layer (see below) samples was determined by precipitation with $10 \%$ trichloroacetic acid (TCA) followed by vacuum filtration (Eldridge et al., 1988). SDS-PAGE and fluorography, pepsin digestion, and sonication, and assay of cell layers for DNA were performed as previously described (Eldridge et al., 1988). Immunoprecipitation of metabolically labeled cell layer samples after $4 \mathrm{~m}$ guanidine extraction was performed as previously described (Eldridge et al., 1986).

Assay for medium-associated proteolytic activity. For each culture, at the end of the $24 \mathrm{hr}$ labeling period, the medium was transferred to a microfuge tube and, in some cases, spun $5 \mathrm{~min}$ at $1000 \times \mathrm{g}$ to remove cell debris (no protease inhibitors were added); the results were the same whether or not the medium was spun. A sample was removed for analysis (" $0 \mathrm{hr}$ " of medium incubation in the absence of cells), and the remainder was returned to the appropriate $\mathrm{CO}_{2}$ atmosphere at $35^{\circ} \mathrm{C} ; 24$ $\mathrm{hr}$ later an identical medium sample was removed for analysis (" $24 \mathrm{hr}$ " of medium incubation in the absence of cells). At both the 0 and $24 \mathrm{hr}$ time points, the medium samples were immediately made to $4 \%$ serum, and aliquots were taken for TCA precipitation and SDS-PAGE, as described above.

Quantitation of myelination, immunochemistry, and electron microscopy. At the end of the 2 week supplementation period, cultures were fixed and processed for Sudan black staining and myelin quantitation or processed for immunocytochemistry (in the living state) with antibodies to basal lamina components as previously described (Eldridge et al., 1987). The following antibodies were used: rabbit antibodies to EHS sarcoma laminin and type IV collagen $\mathrm{NCl}$ domain (from Dr. R. Timpl; Eldridge et al., 1987), mouse monoclonal antibody to basal lamina HSPG (from Dr. C. Cornbrooks; Eldridge et al., 1986, 1987), rabbit antibodies to EHS sarcoma and to human placental type IV collagen (from Dr. E. Crouch), and rabbit antibodies to EHS sarcoma type IV collagen $\mathrm{NCl}$ domain (from Dr. A. Charonis). All antibodies were specific for the stated antigen, as determined by immunoprecipitation of medium or cell-layer samples from metabolically labeled, differentiated SC-neuron cultures. Some cultures were fixed and embedded for electron microscopy; fascicles were transversely sectioned, stained in thin sections with uranyl acetate and lead citrate, and examined in a Philips 300 EM as previously described (Bunge et al., 1980; Ratner et al., 1986). 
Table 1. Assay for medium-associated proteolytic activity

\begin{tabular}{|c|c|c|c|c|}
\hline Experiment & Additions to DM & $\begin{array}{l}\text { TCA precipitable } \\
\frac{\text { cpm at } 24 \mathrm{hr}}{\text { TCA precipitable }} \times 100^{a} \\
\text { cpm at } 0 \mathrm{hr}\end{array}$ & SEM & $\begin{array}{l}\text { Basal lamina } \\
\text { and myelin } \\
\text { formation }^{\nu} \\
\end{array}$ \\
\hline \multirow[t]{2}{*}{1} & none & 95 & 1.4 & - \\
\hline & $\mathrm{HPS}+\mathrm{EE}(\mathrm{MM})$ & 99 & 1.1 & + \\
\hline \multirow[t]{4}{*}{2} & none & 86 & 0.6 & - \\
\hline & A & 87 & 1.0 & \pm \\
\hline & dFBS & $99^{c}$ & 0.7 & - \\
\hline & $\mathrm{dFBS}+\mathrm{A}(\mathrm{MM})$ & $99^{c}$ & 0.6 & + \\
\hline \multirow[t]{2}{*}{3} & none & 97 & 1.0 & - \\
\hline & $\mathrm{dFBS}+\mathrm{A}(\mathrm{MM})$ & 100 & 0.6 & + \\
\hline
\end{tabular}

SC-neuron cultures, grown in DM for 4 weeks and then in triplicate in the medium indicated for an additional 2 weeks, were metabolically labeled with ${ }^{3} \mathrm{H}$-leucine for $24 \mathrm{hr}$. The culture media were individually assayed for proteolytic activity as described in Materials and Methods. The serum used in experiment 1 was $15 \%$ HPS and in experiments 2 and 3 was $15 \%$ dialyzed FBS (dFBS). A indicates L-ascorbic acid, at $50 \mu \mathrm{g} / \mathrm{ml}(280 \mu \mathrm{M}) ; E E$ indicates $5 \%$ chick embryo extract.

${ }^{a}$ Mean of values from 3 cultures per condition.

"See Eldridge et al. (1987).

${ }^{c} p<0.001$ (2-tailed) compared to DM by $t$ test. All other values are not significantly different from DM.

\section{Results}

The SC-neuron cultures used in this study contained a purified sensory neuron population repopulated with SCs in a defined medium lacking ascorbic acid and serum. In this medium, SCs are unable to deposit basal lamina or form myelin sheaths. In the first paper in this series (Eldridge et al., 1987), we demonstrated that the addition of ascorbic acid to DM, in the presence of a nondialyzable serum component(s), stimulates SC basal lamina assembly and SC myelin formation with similar doseresponse characteristics. These results suggested a model in which the critical need for ascorbic acid did not derive from a direct cffect of that vitamin on SC myclination but, rather, from a direct effect on basal lamina assembly, the latter then enabling the $\mathrm{SC}$ to proceed to myelination. The experiments reported here were performed to test this model.

\section{$S C$ secretion in defined versus supplemented media}

SC-neuron cultures were maintained in DM or supplemented DMs for 2 weeks and then labeled with ${ }^{3} \mathrm{H}$-leucine for $24 \mathrm{hr}$. The SDS-PAGE pattern of labeled proteins in the medium of cultures grown in DM was (with exceptions discussed below) similar to that when cultures were grown in MM (containing ascorbic acid and serum); many bands representing proteins ranging in size from $M_{r}<20,000$ to $>200,000$ were present (cf. Fig. 1, $a, e$ ). Similar results were obtained by 2 additional protocols for labeling cultures grown in DM (cf. Fig. 1, $a-c$ ), including the procedure used initially in this laboratory (Fig. $1 b$; Carey and Bunge, 1981). Culture media were also analyzed after an additional $24 \mathrm{hr}$ of incubation in the absence of cells. There was little or no loss of TCA-precipitable cpm (Table 1) or degradation of protein (Fig. $1, d, f$ ) during this time period, indicating that the accumulation of secreted proteins had not been affected by proteolysis.

The relative amount of protein synthesized and secreted by SCs in DM and MM was quantitated after ${ }^{3}$ II-leucine labeling by TCA precipitation of medium and cell layer samples, followed by normalization of TCA-precipitable $\mathrm{cpm}$ to culture DNA content. As shown in Table 2, no systematic differences in the quantity of protein synthesized or secreted were found.
Five experiments were analyzed in this way, and although there were often very significant differences in total protein synthesis and/or secretion within a given experiment, the lack of a consistent correlation between the state of SC differentiation and these aspects of protein biosynthesis suggests that such quantitative differences are not a critical factor controlling SC differentiation.

\section{Ascorbic acid is required for the formation of triple-helical collagen}

Although the SDS-PAGE patterns of proteins secreted by SCs grown with neurons in DM and in MM were similar, some diffcrences were observed, especially in the $M_{r}=170,000$ 200,000 region, where procollagens produced by SCs are known to migrate (Bunge et al., 1983; Carey et al., 1983). Whereas the procollagen type IV doublet and other bands known to be collagenase-sensitive were apparent in the medium of cultures in MM (Fig. 2, $g, h$ ), only broad, poorly defined bands were observed in the same region when medium from DM cultures was analyzed (Fig. 2, $a, b$ ). To determine whether SCs in DM were capable of synthesizing and secreting native, triple-helical collagens, metabolically labeled medium samples were digested with pepsin, which cleaves essentially all proteins, including non-triple-helical collagen domains, but is inactive against triple-helical collagen domains. Whereas pepsin-resistant bands known to correspond to collagen types I and IV (Carey et al., 1983) were observed in the medium of cultures grown in MM (Fig. 2, $o, p$ ), little, if any, pepsin-resistant collagen was secreted by cultures grown in DM (Fig. $2, i, j$ ). Identical results were obtained when cell layers were examined for pepsin-resistant collagens (data not shown).

As discussed above, MM differs from DM in that it contains ascorbic acid and serum. To determine the individual effects of these additives on SC biosynthetic processes, cultures were grown and labeled with ${ }^{3} \mathrm{H}$-leucine in DM supplemented with only ascorbic acid or serum and then analyzed as above. Paralleling the lack of detectable effects on SC differentiation, serum alone did not alter the SDS-PAGF pattern of either total medium proteins (Fig. 2,e,f) or pepsin-resistant collagens (Fig. 2, $m$, $n$ ). Proteins secreted by cultures grown in DM supplemented 


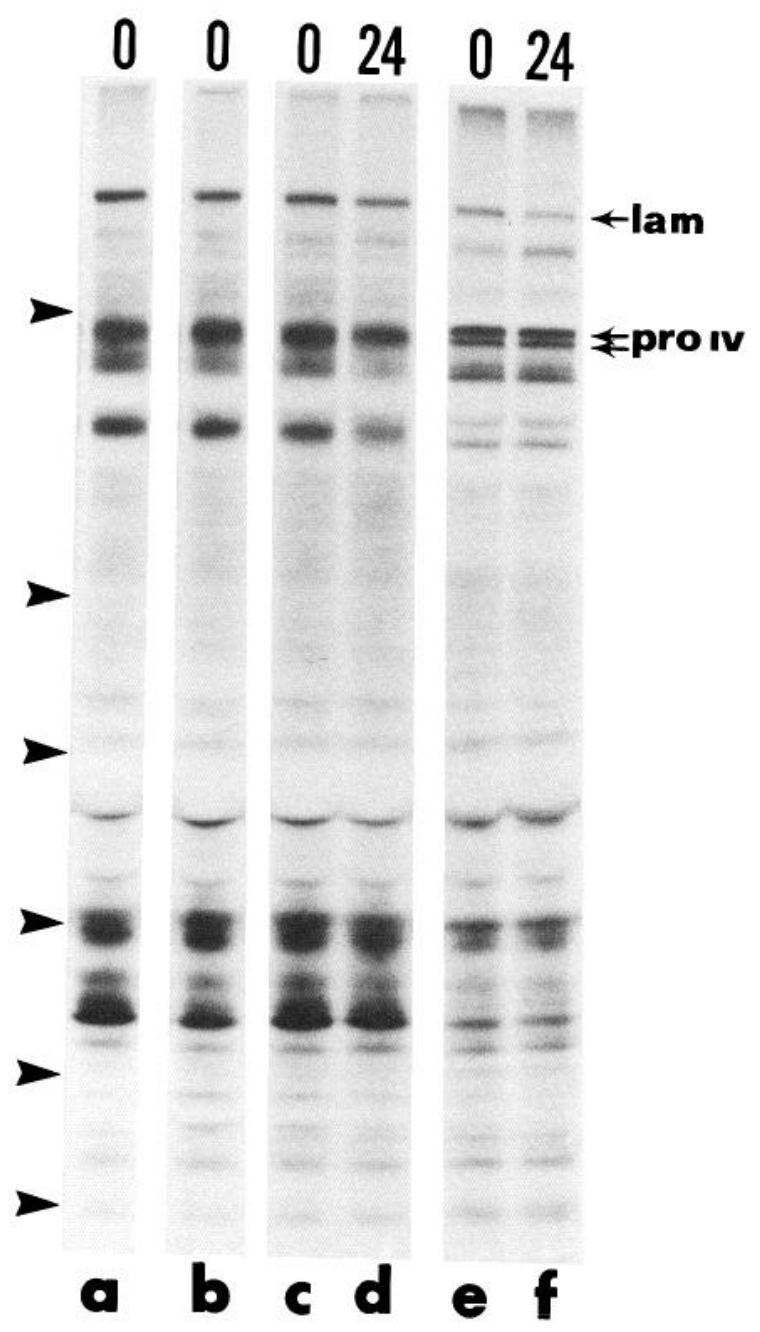

Figure 1. Effects of different growth and labeling media on the pattern of proteins secreted by SCs cultured with neurons. SC-neuron cultures were grown for 4 weeks in DM and then for 2 weeks in DM (lanes $a$ d) or MM ( $15 \%$ dialyzed FBS plus ascorbic acid; lanes $e$ and $f)$. The cultures were then labeled with ${ }^{3} \mathrm{H}$-leucine and, in some cases, assayed for medium-associated proteolytic activity as described in Materials and Methods. For $0 \mathrm{hr}$ samples, each lane was loaded with equal TCAprecipitable medium cpm; for $24 \mathrm{hr}$ samples, the lanes were loaded with volumes of medium equal to the corresponding $0 \mathrm{hr}$ samples. The samples were subjected to SDS-PAGE on a linear 5-10\% gel followed by fluorography. Lane $a, 0 \mathrm{hr}$ sample from a DM culture labeled in DM; lane $b, 0 \mathrm{hr}$ sample from a DM culture labeled in leucine-free Eagle's minimal essential medium plus NGF, as described by Carey and Bunge (1981); lanes $c$ and $d, 0(c)$ and $24(d) \mathrm{hr}$ samples from a DM culture labeled in leucine-free Eagle's minimal essential medium containing 5.2 $\mu \mathrm{g} / \mathrm{ml} \mathrm{L}$-leucine plus normal DM components; lanes $e$ and $f, 0(e)$ and $24(f) \mathrm{hr}$ samples from a culture grown for 2 weeks in MM and then labeled in leucine-free Eagle's minimal essential medium containing 5.2 $\mu \mathrm{g} / \mathrm{ml} \mathrm{L}$-leucine plus normal DM components, $5 \%$ dialyzed FBS, and $50 \mu \mathrm{g} / \mathrm{ml}$ ascorbic acid. The positions of some identified bands are indicated (see Cornbrooks et al., 1983; Carey et al., 1983). Lam, laminin A chain; pro IV, type IV procollagen. Arrowheads indicate the positions of molecular-weight standards, myosin $\left(M_{r}=200,000\right)$, phosphorylase B $\left(M_{r}=97,000\right)$, BSA $\left(M_{r}=69,000\right)$, ovalbumin $\left(M_{r}=46,000\right)$, carbonic anhydrase $\left(M_{r}=30,000\right)$, and lactoglobulin $\mathrm{A}\left(M_{r}=18,000\right)$.

Figure 2. SDS-PAGE patterns of total protein and triple-helical collagen secreted by SCs grown in different media. SC-neuron cultures grown for 4 weeks in DM and then for 2 weeks in various supplemented DMs were labeled with ${ }^{3} \mathrm{H}$-leucine as described in Materials and Methods. All samples were made to $4 \%$ FBS at the end of the labeling period.
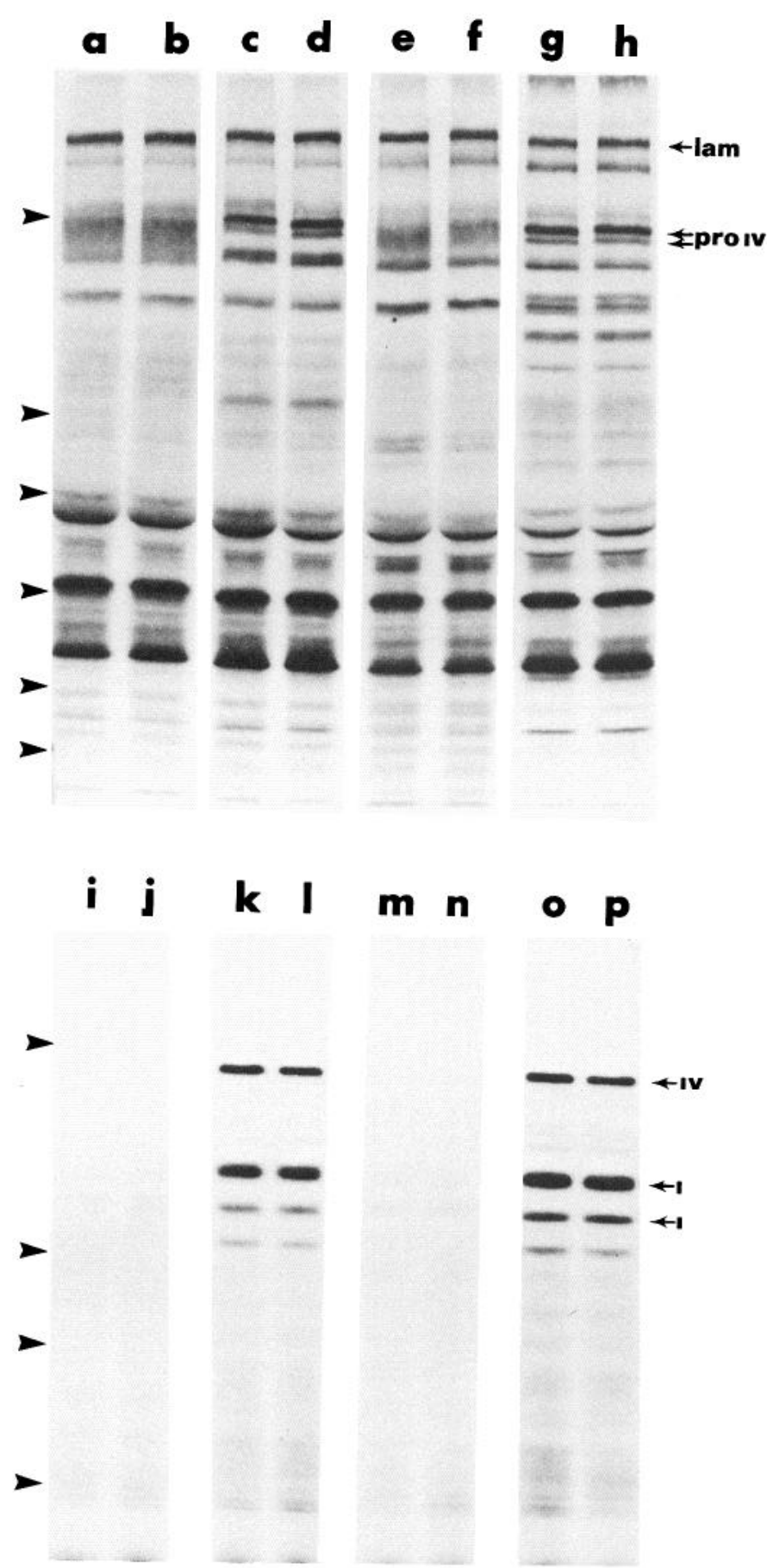

Samples were subjected to SDS-PAGE on linear 5-10\% (lanes $a-h$ ) or $6 \%$ (lanes $i-p$ ) gels followed by fluorography. Each lane represents equal TCA-precipitable medium cpm; counts were determined prior to pepsin digestion for lanes $i-p$. Lanes $a$ and $b$, whole media, and $i$ and $j$, pepsindigested media, from 2 different cultures grown in DM. Lanes $c$ and $d$, whole media, and $k$ and $l$, pepsin-digested media, from 2 different cultures grown in DM supplemented with ascorbic acid. Lanes $e$ and $f$, whole media, and $m$ and $n$, pepsin-digested media, from 2 different cultures grown in DM supplemented with dialyzed FBS. Lanes $g$ and $h$, whole media, and $o$ and $p$, pepsin-digested media, from 2 different cultures grown in DM supplemented with ascorbic acid and dialyzed FBS (MM). The positions of some identified bands are indicated (see Bunge et al., 1983; Carey et al., 1983; Cornbrooks et al., 1983). Lam, laminin A chain; pro $I V$, type IV procollagen; $I V$, type IV collagen; $I$, $\alpha-1$ (upper) and $\alpha$-2 (lower) chains of type I collagen; band beneath lower $I$ probably corresponds to $\alpha-2$ (IV) $-70 \mathrm{~K}$. Arrowheads indicate the positions of molecular-weight standards, myosin $\left(M_{r}=200,000\right)$, phosphorylase B $\left(M_{r}=97,000\right)$, BSA $\left(M_{r}=69,000\right)$, ovalbumin $\left(M_{r}=\right.$ $46,000)$, carbonic anhydrase $\left(M_{r}=30,000\right)$, and lactoglobulin A $\left(M_{r}=\right.$ 18,000 ); in lanes $i-p$, the 2 lowest $M$, standards are not present. 
Table 2. Synthesis and secretion of protein by SCs grown with neurons in MM compared with DM

\begin{tabular}{|c|c|c|c|}
\hline & Components & $\frac{\mathrm{cpm} / \mu \mathrm{g} \text { DNA of cultures in } \mathrm{MM}^{a}}{\mathrm{cpm} / \mu \mathrm{g} \text { DNA of cultures in DM }}$ & \\
\hline Experiment & of $\mathrm{MM}$ & Protein synthesized $(M+C L)$ & Protein secreted $(\mathrm{M})$ \\
\hline 1 & HPS + EE & $\frac{4.78 \times 10^{5}}{4.77 \times 10^{5}}: 100 \%$ & $\frac{5.16 \times 10^{4}}{3.95 \times 10^{4}}: 130 \%$ \\
\hline 2 & $\mathrm{HPS}+\mathrm{EE}$ & $\frac{1.24 \times 10^{6}}{1.68 \times 10^{6}}: 74 \%$ & $\frac{1.43 \times 10^{5}}{1.79 \times 10^{5}}: 80 \%$ \\
\hline 3 & $\mathrm{dFBS}+\mathrm{A}$ & $\frac{1.13 \times 10^{6}}{0.93 \times 10^{6}}: 122 \%^{c}$ & $\frac{1.24 \times 10^{5}}{1.64 \times 10^{5}}: 76 \%^{i}$ \\
\hline 4 & $\mathrm{dFBS}+\mathrm{A}$ & $\frac{1.74 \times 10^{6}}{1.09 \times 10^{6}}: 160 \%^{d}$ & $\frac{2.46 \times 10^{5}}{1.51 \times 10^{5}}: 163 \%^{d}$ \\
\hline 5 & $\mathrm{dFBS}+\mathrm{A}$ & $\frac{1.02 \times 10^{6}}{0.99 \times 10^{6}}: 103 \%$ & $\frac{1.07 \times 10^{5}}{0.64 \times 10^{5}}: 166 \% \%^{d}$ \\
\hline
\end{tabular}

Average

$112 \%$

$123 \%$

SC-neuron cultures, grown in DM for 4 weeks and then in triplicate in cither DM or in MM for an additional 2 weeks, were metabolically labeled with ${ }^{3} \mathrm{H}$-leucine for $24 \mathrm{hr}$. The culture media (M) and cell layers (CL) were individually assayed for TCA-precipitable cpm and DNA as described in Materials and Methods. MM was 25\% HPS plus 10\% EE in Experiment 1, 15\% HPS plus 5\% EE in Experiment 2, and 15\% dFBS plus $50 \mu \mathrm{g} / \mathrm{ml}$ ascorbic acid in Experiments 35. Abbreviations are given in Table 1.

${ }^{a}$ Means of values from 3 cultures per condition; number after colon is ratio of the two values, times $100 \%$.

" $p<0.01$.

${ }^{c} p<0.05$ (2-tailed) compared with DM by $t$ test.

${ }^{d} p<0.005$.

with ascorbic acid alone (Fig. 2, c, d) were similar to those secreted by cultures given ascorbic acid plus serum (MM; Fig. $2, g, h)$. In addition, the pepsin-resistant collagens observed in the medium of cultures grown in DM supplemented with ascorbic acid alone were also similar to those in the medium of cultures grown in MM (cf. Fig. 2, $k$ and $l$ with $o$ and $p$ ).

Taken together, these results indicate that, whereas SCs grown on axons in DM are able to synthesize and secrete an array of proteins similar to that produced by fully differentiated SCs in $\mathrm{MM}$, they are unable to produce significant amounts of the native, triple-helical collagens produced by SCs in MM. Furthermore, the ascorbic acid component of MM can account for the effects of MM on promoting the biosynthesis of triple-helical collagens, suggesting that triple-helical type IV (basement membrane) collagen production might mediate the effects of ascorbic acid on basal lamina assembly.

\section{ODCs do not require ascorbic acid to form myelin}

The ODC forms myelin in CNS tissues without the concomitant deposition of a basal lamina (Peters et al., 1976). This fact suggested that an examination of the influence of ascorbic acid on ODC myelination would reveal whether ascorbic acid plays a role in myelin formation per se, independent of its effects on basal lamina formation. Wood and Williams (1984) have shown that ODCs from embryonic spinal cord will interact with dorsal root ganglion cell axons in our culture system, initially proliferating and then forming myelin, and that such ODCs do not possess basal lamina. We therefore examined the requirements of ODC myelin formation for culture medium components previously shown to be required for SC myelin formation.

Suspensions of cells dissociated from embryonic day 15 spinal cord (containing ODC precursors) were plated onto cultures of purified dorsal root ganglion neurons. The cultures were maintained first in DM (2 weeks) and then in DM or various supplemented DMs (4 weeks) before being analyzed for myelin formation. As shown in Table 3, in DM or DM supplemented with serum, ODCs formed $80-90 \%$ of the amount of myelin formed in DM supplemented with ascorbic acid and serum. In contrast, SCs exhibited an essentially absolute requirement for ascorbic acid (Table 3 ). These results suggest that the dependence of SC myelination upon ascorbic acid is not due to a direct role of ascorbic acid in the biosynthesis or assembly of

Table 3. Comparison of the requirements for myelination of dorsal root ganglion neurons by oligodendrocytes (ODCs) and Schwann cells (SCs)

\begin{tabular}{|c|c|c|c|c|}
\hline \multirow[b]{3}{*}{ Additions to DM } & \multicolumn{4}{|c|}{ Percent of maximum myelination } \\
\hline & \multicolumn{2}{|l|}{ ODCs } & \multicolumn{2}{|l|}{$\mathrm{SCs}$} \\
\hline & $\begin{array}{l}\text { Aver- } \\
\text { age }^{a}\end{array}$ & SEM & $\begin{array}{l}\text { Aver- } \\
\text { age }^{a}\end{array}$ & SEM \\
\hline None & 81 & 6.1 & 0 & 0 \\
\hline FBS & 88 & 12 & 0.0009 & 0.0007 \\
\hline dFBS & 88 & 8.2 & 0.0003 & 0.0003 \\
\hline $\mathrm{dFBS}+\mathrm{A}(\mathrm{MM})$ & 100 & 6.1 & 100 & 6.3 \\
\hline
\end{tabular}

CNS glial cell-neuron cultures were grown in DM for 2 weeks and then in duplicate in the medium indicated for an additional 4 weeks. Abbreviations are given in Table 1; ascorbic acid was used at $50 \mu \mathrm{g} / \mathrm{ml}$, and dFBS at $15 \%$. For analysis, culturcs were stained with antibody to $P_{0}$ to detect colonies of myclin-forming SCs, fixed, and stained with Sudan black, as described in Materials and Methods. ODC myelin formation was assessed by determination of the percentage of fields $\left(0.024 \mathrm{~mm}^{2}, 240\right.$ fields/culture) containing ODC myelin. An average of $8.5 \%$ of the fields in cultures grown in MM contained SC myelin and thus were counted as not containing ODC myelin; it was assumed that a similar proportion of fields in cultures grown in non-ascorbic acid-supplemented media contained non-myelinforming SCs. The experiment was performed twice; the percentage of fields containing ODC myelin in MM was $44 \%$ in one experiment and $70 \%$ in the other.

SC-neuron cultures were grown for 4 weeks in DM and then in duplicate in the medium indicated for an additional 2 weeks. SC myelin formation was quantitated after fixation and Sudan black staining by counting myelin segments (Eldridge et al. 1987). The experiment was performed twice; SC myelin segments $/ \mathrm{mm}^{2}$ in MM were 314 in one experiment and 279 in the other.

a Average of values from duplicate cultures from duplicate experiments. 

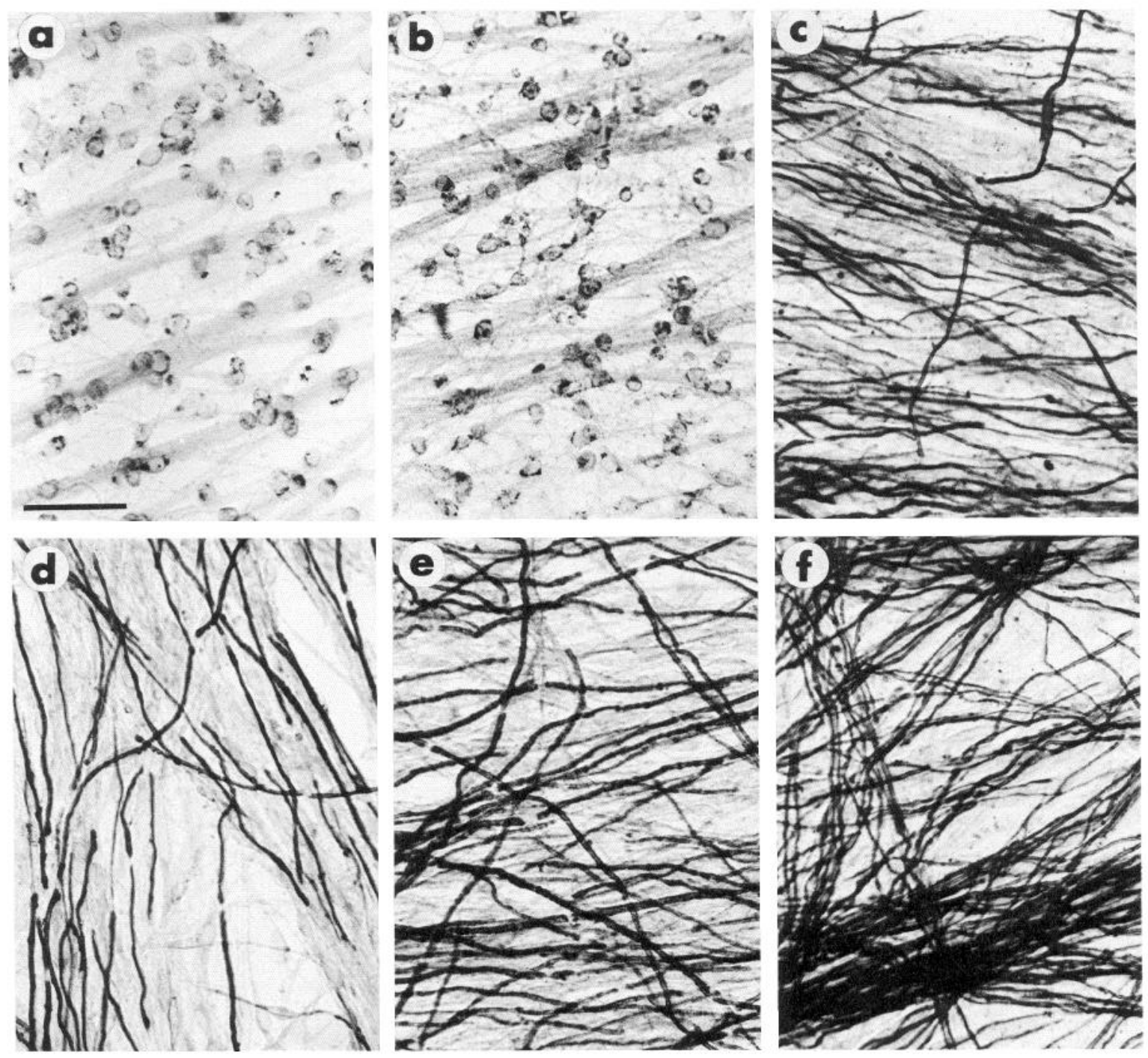

Figure 3. Effects of BLG and FCG overlays on myelin formation by SCs grown with neurons in DM or MM (15\% dialyzed FBS plus ascorbic acid). SC-neuron cultures grown for 4 weeks in DM were given no overlays $(a$ and $d)$, FCG overlays $(b$ and $e$ ), or BLG overlays $(c$ and $f)$ and then maintained in DM $(a-c)$ or MM $(d-f)$ for 2 weeks. The cultures were then fixed and stained with Sudan black. In DM, BLG promotes myelin formation, whereas FCG does not. In MM, both BLG and FCG enhance myelin formation. Scale bar, $50 \mu \mathrm{m}$.

myelin lipids and proteins which SCs and ODCs have in common.

\section{Exogenous basal lamina substitutes for ascorbic acid-stimulated basal lamina production by SCs}

Our earlier work (Eldridge et al., 1987) demonstrated that the promotion of SC basal lamina assembly by ascorbic acid is closely correlated with the stimulation of SC myelin formation. In order to determine if there is a causal link between SC basal lamina assembly and myelin formation, we provided exogenous basal lamina to SCs grown with neurons under media conditions in which the SCs are unable to assemble basal lamina of their own production, and we then determined the effect on myelin formation.

SC-neuron cultures that had been maintained in DM were overlaid with either BLG, prepared from an extract of EHS sarcoma basal lamina matrix (Kleinman et al., 1986), or FCG, prepared from an extract of type I collagen-rich rat-tail tendon (Elsdale and Bard, 1972), and were then maintained in DM or supplemented DMs for 2 weeks. Myelin formation was quantitated after staining the cultures with Sudan black. In SC-neuron cultures overlaid with BLG and maintained in DM, myelin was formed in quantities equal to those observed for control cultures given MM but no BLG (Table 4, cf. Fig. 3, c, d). Similar results were obtained when cultures overlaid with BLG were maintained in DM supplemented with serum (but without ascorbic acid; Table 4). The ability of BLG to promote myelin formation by SCs in the absence of ascorbic acid was due specifically to the presence of basal lamina components and not simply to the presence of a gel overlay or extracellular matrix in general, as evidenced by the inability of FCG to promote myelin formation (Table 4) or even the required precedent morphological changes in the SCs (Fig. 3b). The enhancement of myelin formation by BLG and FCG overlays in the presence of ascorbic acid (Table 4) is not understood, but it may be related to an earlier onset of myelination.

Electron microscopic examination of cultures grown in DM and fixed immediately after receiving BLG revealed that the 

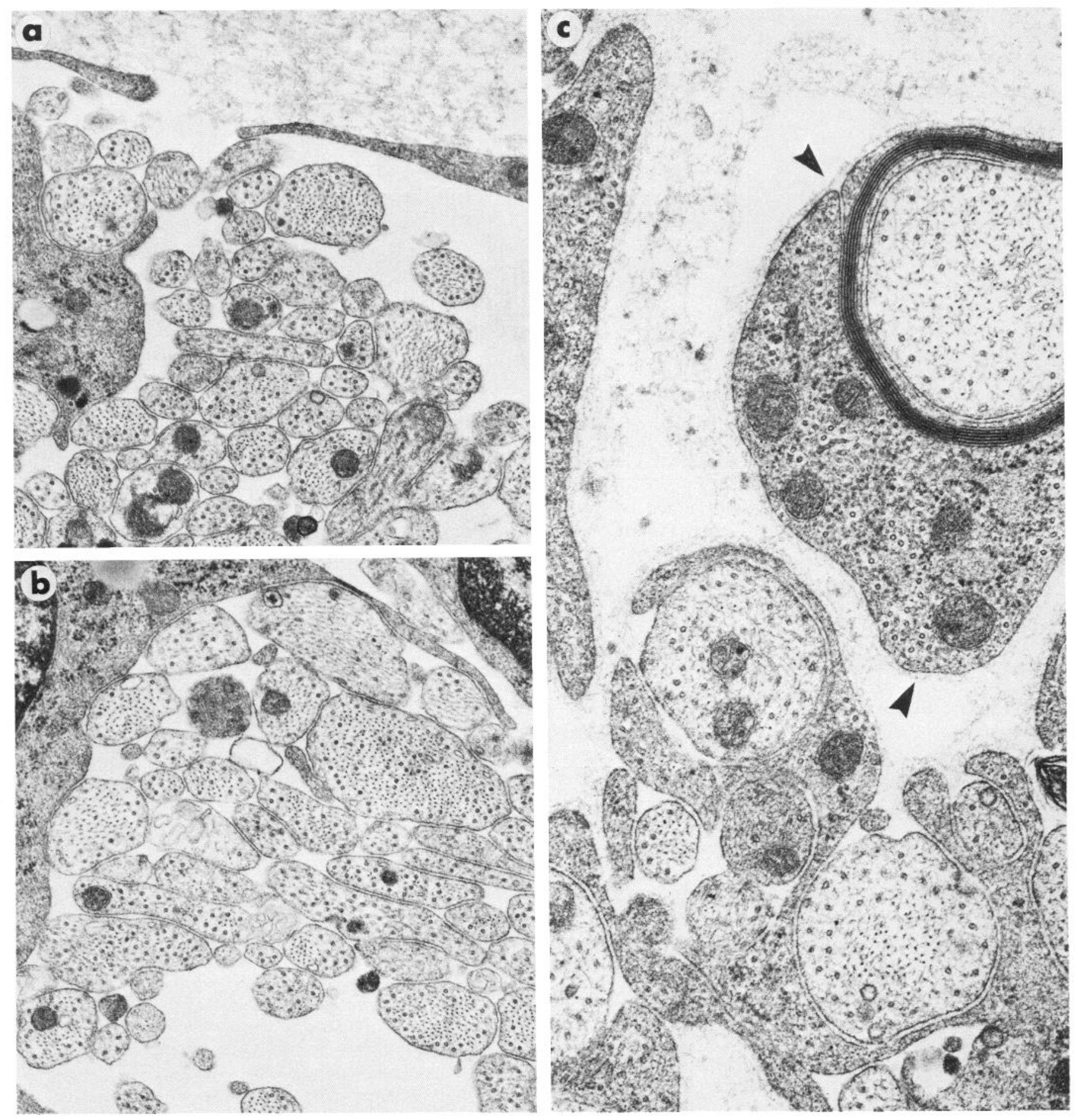

Figure 4. Electron micrographs of cultures in DM within $20 \mathrm{~min}$ of $(a, b)$ and $14 \mathrm{~d}$ after $(c)$ the addition of BLG. $a$, Flattened SC processes facing the medium are covered by BLG matrix that has not permeated the subjacent fascicle of axons. $b$, An area just above the substratum; matrix is not seen in or around this fascicle of axons. The lack of ensheathment of axons is typical of growth in DM. $c$, Two weeks after BLG addition, matrix is seen beneath the culture surface; this material fills spaces between axon-SC units and also may be observed to cover the SC surface in a lamina densa-like manner (arrowheads). In the 2-week period following the addition of BLG to a culture maintained in DM, myelin has formed and unmyelinated axons have become ensheathed with SC cytoplasm. $a$ and $b, \times 24,000 ; c, \times 38,500$.

BLG matrix was initially distributed as a thick layer draped over the surfaces of the tissue (Fig. $4 a$ ). Little matrix was seen on SC surfaces not immediately adjacent to the BLG layer, and none was observed within axon-SC fascicles (Fig. 4, $a, b$ ). In sibling cultures overlaid with BLG and then maintained in DM for 2 weeks before fixation, a lamina densa-like layer had ap- peared on most abaxonal SC surfaces, including those deep within fascicles (Fig. 4c). Accumulations of similar matrix material were also seen away from SC surfaces, filling extracellular spaces between neighboring SCs (Fig. 4c).

The apparent redistribution of BLG matrix in this experiment suggested that the gel property might be unimportant in the 
Table 4. Exogenous basal lamina matrix promotes Schwann cell myelination in the absence of ascorbic acid, but fibrous collagen matrix does not

\begin{tabular}{|c|c|c|c|c|}
\hline \multirow[b]{2}{*}{ Additions to DM } & \multirow[b]{2}{*}{ Overlay: } & \multicolumn{3}{|c|}{ Myelin segments $/ \mathrm{mm}^{2}$} \\
\hline & & None & $\mathrm{FCG}$ & BLG \\
\hline None & & 0 & 0 & 150 \\
\hline drBS & & 0 & 0 & 140 \\
\hline $\mathrm{dFBS}+\mathrm{A}(\mathrm{MM})$ & & 120 & 210 & 300 \\
\hline
\end{tabular}

SC-neuron cultures grown in DM for 4 weeks were given no overlay or FCG or BLG overlays (sęe Materials and Methods) and then maintained in DM or DM supplemented with $15 \%$ dialyzed FBS with or without $50 \mu \mathrm{g} / \mathrm{ml}$ ascorbic acid for 2 weeks. Cultures were fixed and stained with Sudan black, and the amount of myelin which had formed was quantitated (Eldridge et al., 1987). The values given are the averages from duplicate cultures from a representative experiment. Abbreviations are given in Table 1.

effect of BLG on SC myelination. To test this idea we diluted BLG extract in DM so that it could not gel and determined the ability of such diluted extract to promote myelin formation in the absence of ascorbic acid. As shown in Table 5, it promoted myelination comparable to that observed in $\mathrm{MM}$, both in the presence and absence of serum and even at dilutions of 1:501:100; the addition of diluted matrix also led to the deposition on SC surfaces of laminin, type IV collagen, and HSPG, as determined by immunofluorescence (data not shown).

\section{Laminin promotes SC myelin formation in the absence of ascorbic acid}

To determine whether any single component could substitute for the complex mixture present in the extract of basal lamina matrix, we tested 3 of the major known basal lamina molccules for their ability to promote $\mathrm{SC}$ myelination in the absence of ascorbic acid. Laminin at $50 \mu \mathrm{g} / \mathrm{ml}\left(50 \mathrm{nM}\right.$, assuming $10^{6} \mathrm{gm} /$ mol; Timpl et al., 1979), type IV collagen at $27 \mu \mathrm{g} / \mathrm{ml}(50 \mathrm{nM}$, assuming $5.4 \times 10^{5} \mathrm{gm} / \mathrm{mol}$; Kleinman et al., 1982), and basal lamina HSPG at $3.75 \mu \mathrm{g} / \mathrm{ml}\left(5 \mathrm{nM}\right.$, assuming $7.5 \times 10^{5} \mathrm{gm} /$ mol; Ledbetter et al., 1985) were tested, both singly and in combination. As shown in Table 6 (Part 1), 50 nм laminin, in

\section{Table 5. Diluted, non-gel-forming basal lamina matrix promotes} Schwann cell myelination in the absence of ascorbic acid

\begin{tabular}{lc} 
Additions to DM & $\begin{array}{c}\text { Myelin } \\
\text { segments } / \mathrm{mm}^{2}\end{array}$ \\
\hline Experiment 1 & 0 \\
None & 140 \\
dFBS + A (MM) & 160 \\
1:50 basal lamina matrix & 120 \\
1:200 basal lamina matrix & 92 \\
1:500 basal lamina matrix & \\
Experiment 2 & 0 \\
dFBS & 320 \\
dFBS + A (MM) & 330 \\
dFBS + 1:20 basal lamina matrix & 280 \\
dFBS + 1:100 basal lamina matrix & 120 \\
dFBS + 1:500 basal lamina matrix & \\
\hline SC-neuron cultures grown in DM for 4 weeks were maintained for an additional \\
2 weeks in the medium indicated and then processed for myelin quantitation \\
(Eldridge et al., 1987). The values given are the averages of duplicate cultures \\
from representative experiments. Ice-cold basal lamina matrix was diluted into \\
ice-cold DM (Experiment 1) or DM supplemented with $15 \%$ dialyzed FBS \\
(Experiment 2). Ascorbic acid was used at 50 $\mu$ g/ml. Abbreviations are given in \\
Table 1.
\end{tabular}

Table 6. Laminin promotes Schwann cell myelination in the absence of ascorbic acid

1. Representative experiment

\begin{tabular}{lcc}
\hline & \multicolumn{2}{c}{ Myelin segments $/ \mathrm{mm}^{2}$} \\
\cline { 2 - 3 } Additions to medium & $\begin{array}{l}\text { Without } \\
\text { dFBS }\end{array}$ & $\begin{array}{c}\text { With } \\
\text { dFBS }\end{array}$ \\
\hline None & 0 & 0 \\
A & 29 & 270 \\
10:1:10 nM laminin:HSPG:IV & - & 23 \\
10 nM laminin & - & 27 \\
50:5:50 nM laminin:HSPG:IV & - & 260 \\
50 nM laminin & 180 & 230 \\
5 nM HSPG & - & 0 \\
50 nM IV & - & 0.2
\end{tabular}

2. Summary of 3 experiments

\begin{tabular}{lcc}
\hline & \multicolumn{2}{c}{ Myelin segments $/ \mathrm{mm}^{2}$} \\
\cline { 2 - 3 } Additions to DM & Average & SEM \\
\hline None & 0 & 0 \\
dFBS & 0 & 0 \\
dFBS + A (MM) & 290 & 12 \\
50 nM laminin & 190 & 13 \\
dFBS + 50 nM laminin & 260 & 13
\end{tabular}

SC neuron cultures grown in DM for 4 weeks were maintained for an additional 2 weeks in the medium indicated and then processed for myelin quantitation (Eldridge et al., 1987). The values given are averages (1) from duplicate cultures from a representative experiment or (2) from duplicate cultures from 3 experiments. Ascorbic acid was uscd at $50 \mu \mathrm{g} / \mathrm{ml}$, and dFBS was uscd at $15 \%$. IV indicatcs typc IV collagen; other abbreviations are given in Table 1 . Ice-cold basal lamina components were diluted into ice-cold DM or DM supplemented with dFBS Basal lamina component molar ratios were chosen to reflect the ratios likely to exist in vivo (Kleinman et al., 1983).

the absence of ascorbic acid, promoted myelin formation that was nearly as extensive as that observed in control cultures given MM, but neither type IV collagen nor HSPG had any significant effect on myelination. Neither higher concentrations ( $33 \mathrm{nM}$ ) of HSPG nor a combination of type IV collagen and HSPG (50:5 $\mathrm{nM}$ ) promoted myelination (data not shown); in addition, these 2 molecules did not appear to enhance the effect of laminin significantly, either at high (50 nM) or at suboptimal (10 nM) laminin concentrations (Table 6, Part 1). The effect of laminin addition was observed with laminin obtained from 3 sources (including one noncommercial) and was very consistent from one experiment to another (Table 6, Part 2). The effect of laminin was influenced by serum (Table 6), in contrast to the effect obtained with BLG matrix (Tables 4 and 5).

Examination of cultures by electron microscopy showed that lamina densa-like layers had accumulated on abaxonal SC surfaces in the presence of exogenous laminin (Fig. $5 a$ ) and also that similar-appearing material frequently filled extracellular spaces between SCs (Fig. $5 a$ ), in contrast to basal lamina formed in response to ascorbic acid (Fig. 5b). Surprisingly, when deposition of basal lamina was assessed by immunofluorescence using antibodies to individual basal lamina components, it was found that the addition of exogenous laminin to the culture medium resulted in the deposition on the SC surface of not only laminin but also type IV collagen and HSPG (Fig. $6, f-h$ ); the intensity of antibody staining observed in this case was at least as great as that seen in control cultures in which endogenous basal lamina assembly had been promoted by ascorbic acid (cf. Fig. 6, $f-h$ and $j-l)$. 

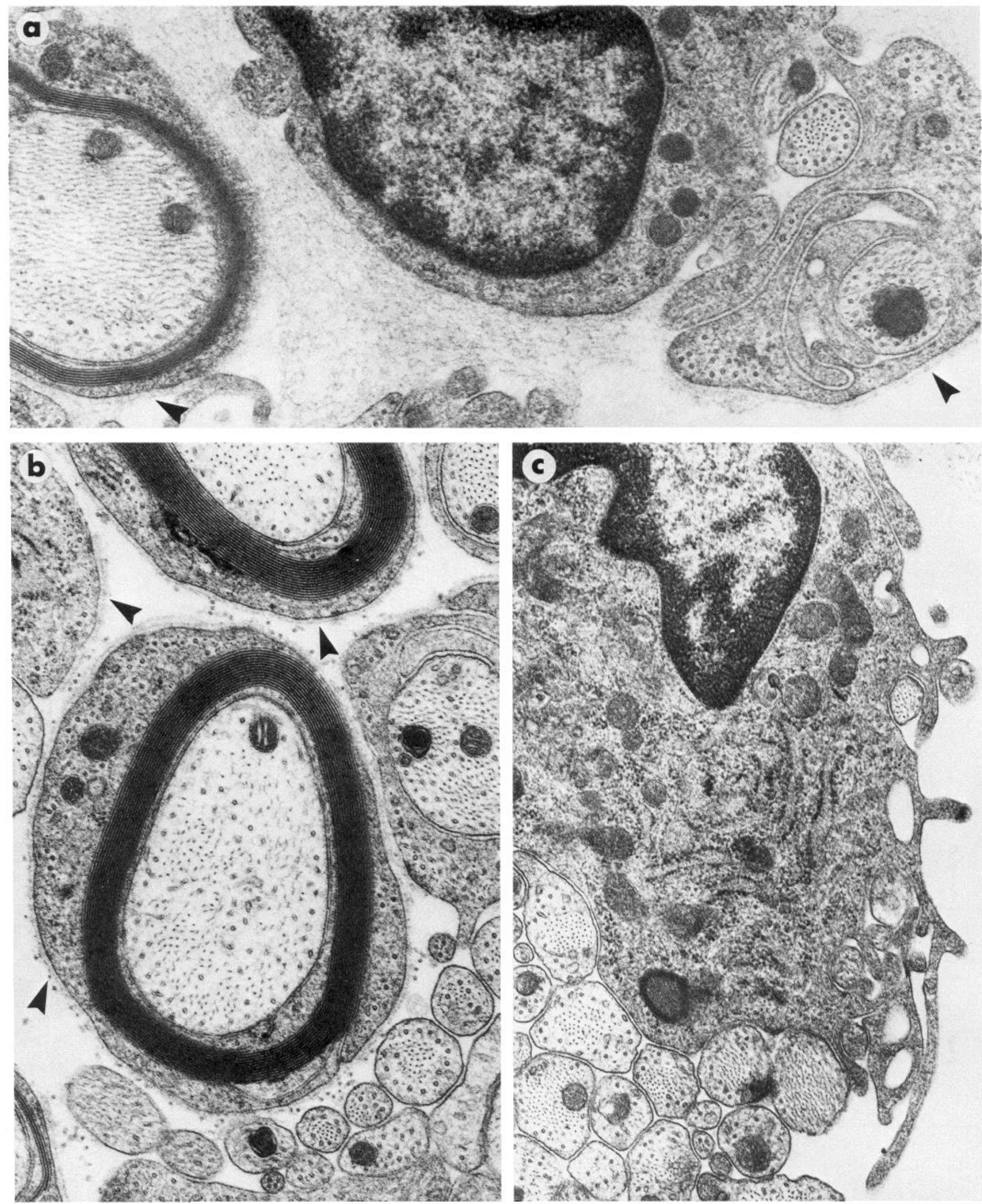

Figure 5. Electron micrographs of cultures grown for 2 weeks in DM supplemented with dialyzed FBS and (a) 50 nM laminin, (b) ascorbic acid $(\mathrm{MM})$, or $(c)$ no other additions. $a$, Matrix has assembled in masses or as a lamina densa-like layer (arrowheads), and ensheathment and myelination of axons have occurred, in the wake of laminin addition. $b$. In cultures grown in medium with ascorbic acid and serum, basal lamina (arrowheads) typically surrounds SCs and slender collagen fibrils have appeared external to the lamina. $c$, Neither extracellular matrix nor ensheathment of axons is apparent in the culture maintained in DM plus serum without additional supplementation. $a$ and $b, \times 38,500 ; c, \times 30,500$. 
LAMININ
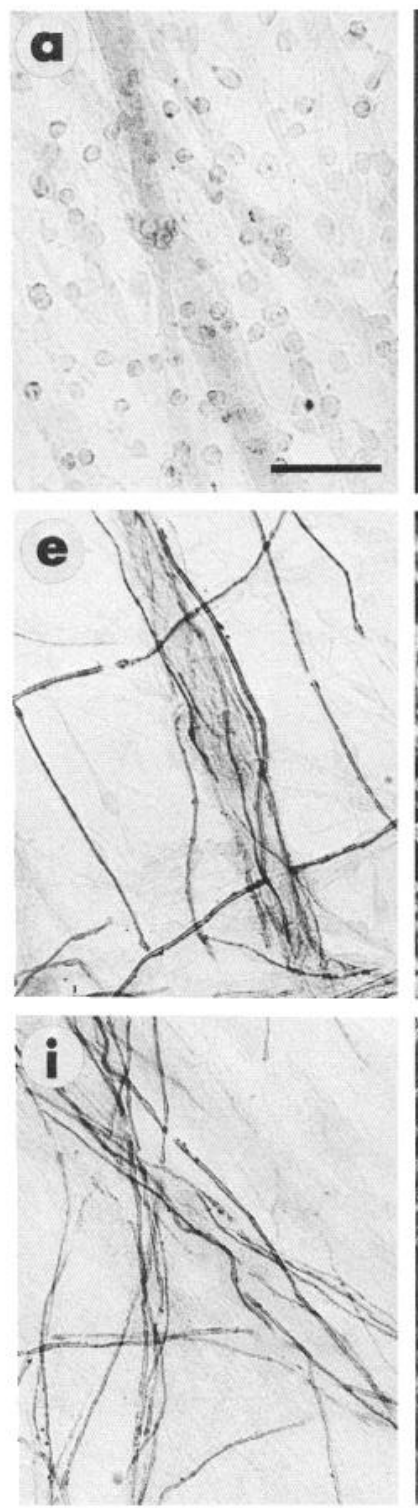
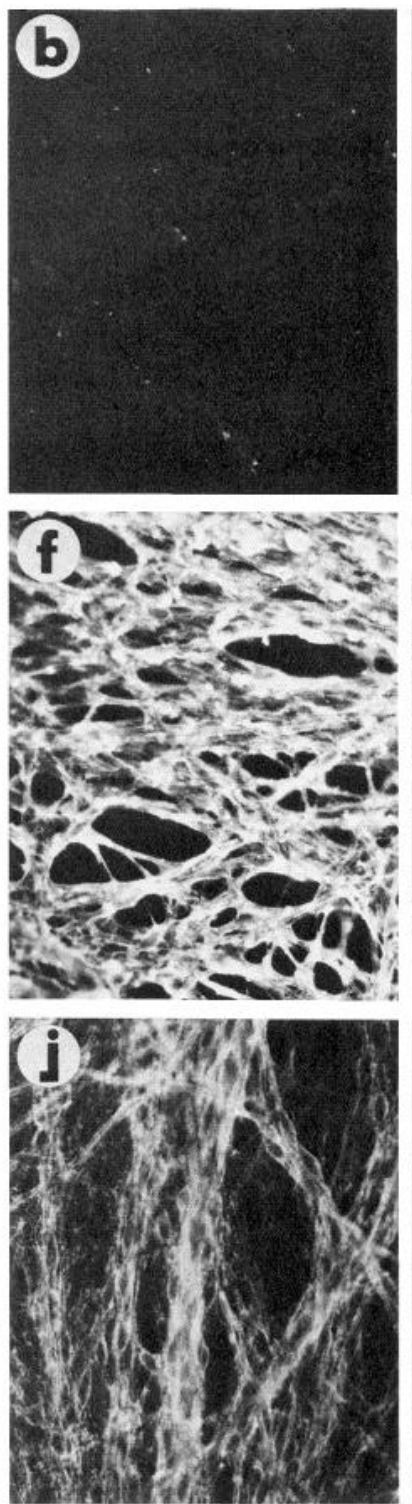
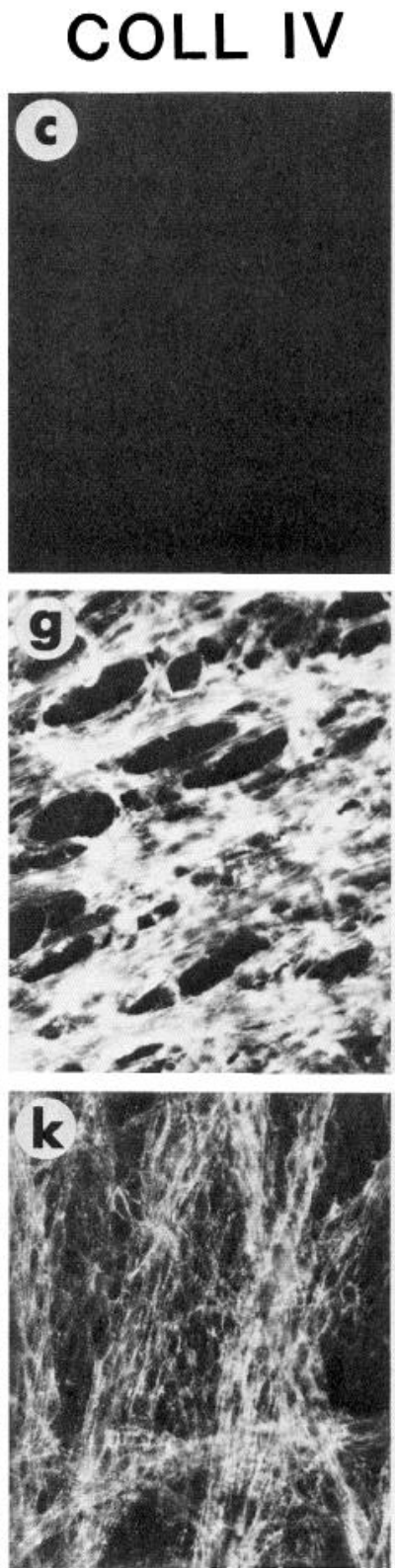

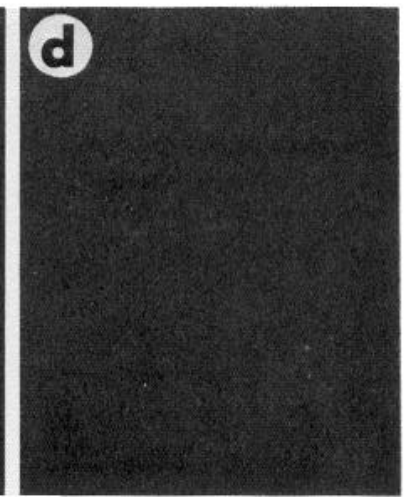

HSPG
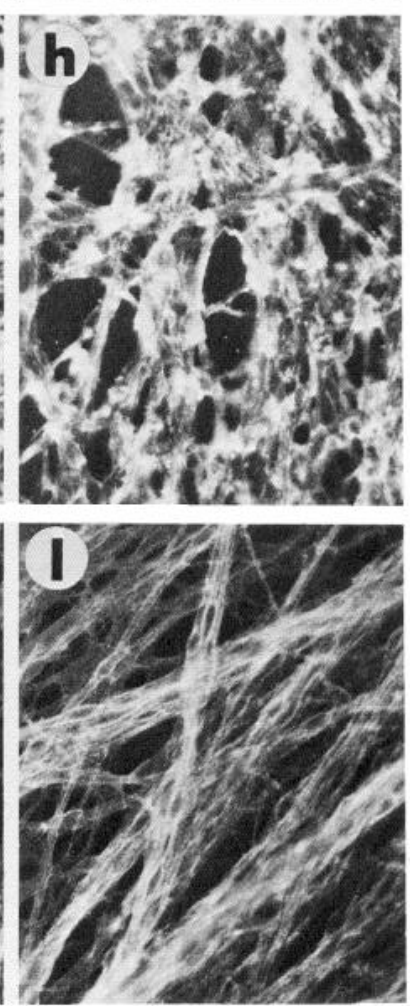

Figure 6. Comparison of basal lamina component deposition by cultures grown for 4 weeks in DM and then 2 weeks in DM supplemented with dialyzed FBS and either no other additions $(a-d), 50$ nм laminin $(e-h)$, or ascorbic acid (MM) ( $i-l)$. Cultures stained with Sudan black $(a, e, i)$, with rabbit anti-laminin followed by fluorescein-conjugated goat anti-rabbit $\operatorname{IgG}(b, f, j)$, with rabbit anti-type IV collagen NCl domain followed by fluorescein-conjugated goat anti-rabbit IgG $(c, g, k)$ or with mouse anti-HSPG followed by rhodamine-conjugated goat anti-mouse IgG ( $d$, $h$, l). Immunocytochemistry was carried out on living cultures. The areas in each column were photographed and printed identically. Scale bar, $50 \mu \mathrm{m}$.

Experiments (not shown) were performed to confirm the apparent deposition of type IV collagen in response to exogenous laminin. First, laminin purified by the method of Timpl et al. (1979) and free of detectable type IV collagen (as determined by both Western blotting and ELISA with polyclonal antibodies to type IV collagen; E. Crouch, personal communication) promoted myelination and the deposition of type IV collagen (as determined by immunofluorescence) comparable with that obtained with commercial laminin preparations. Second, 3 additional antibodies to type IV collagen (one additional antibody to the $\mathrm{NCl}$ domain of EHS sarcoma, one to reduced and alkylated EHS sarcoma, and one to human placental type IV collagen) were all found to stain laminin-induced matrix as described above for the $\mathrm{NCl}$ antibody. These results provide strong evi- dence that the type IV collagen being deposited was derived from SCs and not from contaminants of the laminin preparations.

Attempts to directly demonstrate SC type IV collagen in laminin-induced matrix were only partially successful. Cultures grown in the presence of either ascorbic acid or laminin (with and without serum) were labeled for $24 \mathrm{hr}$ with ${ }^{35} \mathrm{~S}$-methionine, the cell layers were extracted with $4 \mathrm{~m}$ guanidine, and the extracts were dialyzed into immunoprecipitation buffer. All 4 type IV collagen antibodies described above could specifically immunoprecipitate type IV procollagen from extracts of laminintreated cultures (Fig. 7a, lanes 3 and 4 ) but only in small amounts relative to ascorbic acid-treated cultures (Fig. $7 a$, lane 2). The type IV procollagen precipitated from extracts of laminin-treat- 


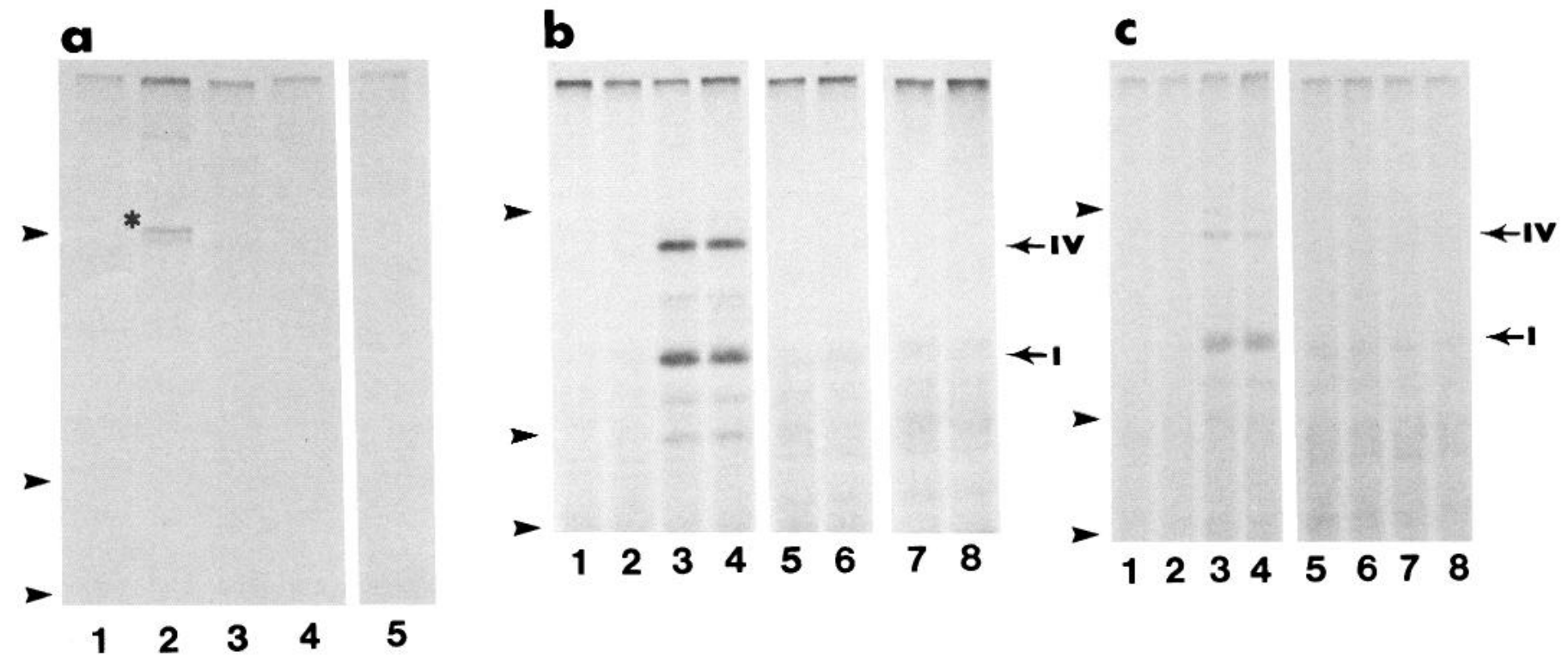

Figure 7. Comparison of collagen deposition promoted by ascorbic acid or exogenous laminin. Cultures grown for 4 weeks in DM and then 1 week in the medium indicated below were labeled for $24 \mathrm{hr}$ in that medium containing $200 \mu \mathrm{Ci} / \mathrm{ml}{ }^{35} \mathrm{~S}$-methionine, and media were then harvested separately from cell layers as described in Materials and Methods. Cell layers were disrupted in $2 \mathrm{ml}$ of PBS in a small-volume homogenizer. Cultures were grown in DM supplemented with dialyzed FBS (lane $1, a$; lanes $1-2, b$ and $c$ ), dialyzed FBS plus ascorbic acid (lanes 2 and 5 , $a$; lanes $3-4, b$ and $c), 50 \mu \mathrm{g} / \mathrm{ml}$ laminin (lane $3, a$; lanes $5-6, b$ and $c$ ), or dialyzed FBS plus $50 \mu \mathrm{g} / \mathrm{ml}$ laminin (lane $4, a$; lanes $7-8, b$ and $c$ ). Arrowheads indicate the positions of molecular-weight standards, myosin $\left(M_{r}=200,000\right)$, phosphorylase B $\left(M_{r}=97,000\right)$, and BSA $\left(M_{r}=69,000\right)$. $a$, Cell layer samples were extracted with $4 \mathrm{~m}$ guanidine, dialyzed into immunoprecipitation buffer, and subjected to immunoprecipitation using rabbit anti-type IV collagen $\mathrm{NCl}$ domain (lanes $1-4$ ) or normal rabbit serum (lane 5), and the precipitates were analyzed by SDS-PAGE on 6\% gels. The asterisk designates the type IV procollagen doublet in lane 2 . Medium $(b)$ and cell layer $(c)$ samples were digested with pepsin and analyzed by SDS-PAGE on $6 \%$ gels. Each pair of lanes $(1-2,3-4$ etc.) represents material from duplicate cultures grown in the same medium. $I V$, type IV collagen; $I$, type I collagen.

ed cultures migrated slightly faster than that of ascorbic acidtreated cultures (Fig. $7 a$, lanes 2 and 3), but the precipitated material was sensitive to bacterial collagenase in both cases (data not shown). Our failure to detect large amounts of SC type IV collagen in these cultures might have been due to an unusual sensitivity of antigenic determinants in non-triple-helical type IV collagen to our extraction procedures. In contrast to ascorbic acid supplementation (Fig. 7, $b$, $c$, lanes 3 and 4), no pepsinresistant collagen was detectable in media (Fig. 7b, lanes 5-8) or cell layers (Fig. 7c, lanes 5-8) of laminin-treated cultures, indicating that the type IV collagen deposited with laminin addition was not triple-helical.

\section{Discussion}

Abundant evidence now exists demonstrating that extracellular matrices are important to cells and tissues, not only for their structural attributes but also for their permissive and even instructive properties with respect to cell proliferation, migration, and differentiation (Kleinman et al., 1981; Sanes, 1983; Hay, 1984; Ekblom et al., 1986). Our results demonstrate a profound requirement of basal lamina matrix for the best-known function of differentiated SCs, myelin formation. Initial work from our laboratory demonstrated that, although SCs can proliferate in the absence of basal lamina (in DM) in response to a mitogen on the surface of axons of dorsal root ganglion neurons, they are unable to differentiate into myelin-forming cells (Moya et al., 1980). Subsequent investigations revealed that SCs require ascorbic acid and a nondialyzable serum factor(s), in addition to axons (Bunge et al., 1982), in order to assemble basal lamina and form myelin; similar dose-response characteristics are observed for the stimulation by ascorbic acid of these 2 aspects of
SC differentiation (Eldridge et al., 1987). Data presented in this paper demonstrate that SCs do not require either ascorbic acid or serum to myelinate axons if they are given basal lamina components from exogenous sources. These data thus support the hypothesis that ascorbic acid acts to enable SCs to form basal lamina and that basal lamina in turn enables SCs to myelinate axons.

In an attempt to determine how ascorbic acid acts to promote SC basal lamina assembly, the synthesis and secretion of protein by SCs grown with neurons in different media were examined. The results demonstrated that, although there were often significant quantitative differences in total protein synthesis and/ or secretion between cultures maintained in different media (and therefore at different stages of differentiation) within an experiment, there was no overall correlation of differences in these general measures of protein biosynthetic processes with stages of SC differentiation. Evaluation of secreted protein by SDSPAGE showed that, with a few exceptions, SCs in DM secreted an array of proteins strikingly similar to that in MM.

Our results contrast significantly with those of Carey and Bunge (1981), who reported that a single protein accounts for essentially all protein secreted by SCs grown with neurons in DM. Because we obtained consistent results using 3 different labeling protocols (including those of Carey and Bunge), we are confident that our results reflect SC protein secretion occurring in DM. Carey and Todd (1987) reported that they observed proteolytic breakdown of protein secreted by SCs in DM. We found no evidence for such breakdown in our cultures, but believe that this discrepancy may be explained by differences in culture technique. Our method of preparing SC-neuron cultures has been modified from that employed by Carey and Bunge 
(1981) and Carcy and Todd (1987); the most significant outcome of these modifications is that we no longer observe the regional degeneration of axons awaiting the slow repopulation of SC.s that occurs in cultures prepared by the older method (Bunge et al,, 1988; C. F. Eldridge and R. P. Bunge, unpublished obser= vations). It is possible that proteases released from degenerating axons (Schlaepfer, 1983) could significantly degrade proteins secreted by SCs in DM and yield the results reported by Carey and Bunge (1981) and Carey and Todd (1987).

Although we observed that SCs in DM exhibited a secretory repertoire similar to that in MM, only SCs in MM were found to synthesize pepsin-resistant, triple-helical collagen; the ascorbic acid component accounted for this effect of MM. A mechanism to account for the effect of ascorbic acid on SC collagen biosynthesis is well established in the literature: Ascorbic acid acts as a cofactor for prolyl hydroxylase which forms, from collagen-bound proline, the hydroxyprolinc residucs essential for the formation and stabilization of the collagen triple-helix (reviewed by Prockop et al., 1976; Kivirikko and Myllyla, 1982). There is evidence suggesting that, at least for interstitial (fibrous) collagens, the lack of a triple-helical conformation increases the susceptibility of collagen chains to degradation intracellularly soon after synthesis (Berg et al., 1980; Neblock and Berg, 1982) and also reduces the rate of secretion of collagen chains, the net result being a severely reduced rate of appearance of functional collagen at the cell surface. Less is known about the role of the triple-helix in type IV collagen secretion and function. Karakashian and Kefalides (1982) reported that the rate of secretion of non-triple-helical type IV collagen in $50 \%$ of that of its fully hydroxylated counterpart, a difference comparable to that observed for type I collagen but that no intracellular degradation is detectable. Kleinman et al. (1983) have shown that denaturation (boiling) of type IV collagen leads to the loss of ability to interact with other basal lamina constituents. It is not clear, however, that denatured type IV collagen is equivalent to nontriple-helical (underhydroxylated) type IV collagen, especially considering that important interactions occur at the globular, noncollagenous C-terminal domain of the type IV collagen molecule (Tsilibary and Charonis, 1986). Our immunofluorescence rcsults (from cultures given exogenous laminin) would suggest that non-triple-helical type IV collagen can interact with other basal lamina components and thus deposit in the matrix. The role of triple-helical collagen in basal lamina assembly is discussed below.

In the experiments reported here we tested the hypothesis that the availability of ascorbic acid leads to SC basal lamina formation, which then leads to myelination. First, we demonstrated that ascorbic acid is unlikely to be directly involved in the biosynthesis and assembly of SC myelin, since myelin formation by ODCs, the non-basal lamina-forming myelinating cell of the CNS, was little affected by ascorbic acid. Second, we provided SCs with an extract of basal lamina matrix in the absence of ascorbic acid and determined whether they could then form myelin. The results of those experiments strongly support our hypothesis, since BLGs promoted control levels of myelination in the absence of ascorbic acid and enabled SCs to acquire basal lamina components on their surfaces. Gels of interstitial collagen (FCGs) did not promote myelination in the absence of ascorbic acid, indicating that the effect of BLGs was specifically due to basal lamina components. Electron microscopic comparison of cultures immediately and 2 weeks after BLG addition suggested that SCs could utilize soluble compo- nents originating from the BLG. Subsequent experiments using BLG matrix diluted so that it could not gel verified that the critical property of the matrix was its content of basal lamina components, not its ability to form a gel. These experiments confirm the original observation made by Carey et al. (1986) that BLGs promote myelin formation by SCs grown with neurons in DM, and they extend it by (1) presenting quantitative data on myelin formation, (2) demonstrating that the effect is specific for BLG, (3) directly demonstrating that non-gel-forming basal lamina extract is fully active in promoting myelination, and (4) illustrating in electron micrographs that the SC surface acquires lamina densa-like structures.

We tested 3 of the major known basal lamina molecules for activity in promoting myelin formation in the absence of ascorbic acid and found that only laminin had any activity, promoting near-control levels of myelination. The ability of laminin to promote myelination was enhanced by serum, in contrast to BLG (from which laminin was purified), suggesting that serum either supplies the $\mathrm{SC}$ with or induces the $\mathrm{SC}$ to synthesize a minor or unknown molecule(s) required for the forming basal lamina structure to acquire full biological activity. Alternatively, considering the relatively impure nature of the BLG (a crude extract), its full myelin-promoting activity in the absence of serum might be due to non-basal lamina contaminants.

We were surprised to find not only that exogenous type IV collagen did not promote myelination, but that provision of exogenous laminin led to bright fluorescence staining for type IV collagen in the absence of ascorbic acid. Since purified laminin known to have no detectable type IV collagen contamination promoted type IV collagen deposition (as well as myelination), the type IV collagen had to originate from SCs. We were unable, however, to directly demonstrate biochemically (by metabolic labeling and immunoprecipitation) the large amounts of SC type IV collagen we expected to see deposited in the matrix in response to exogenous laminin. As mentioned in Results, this result may indicate an exceptional sensitivity of non-triple-helical type IV collagen antigenic determinants to the extraction procedures used.

The apparent equivalence of laminin and ascorbic acid, and nonequivalence of type IV collagen, in promoting basal lamina formation and myelination is puzzling. Exogenous laminin bound abundantly and rapidly $(<1 \mathrm{hr})$ to the surface of SCs grown with neurons in DM, indicating a large number of unoccupied laminin binding sites (a result also obtained with purified SCs by McGarvey et al., 1984), whereas barely detectable amounts of exogenous type IV collagen bound during 2 weeks of exposure, as determined by immunofluorescence (data not shown). It may be that, by binding to laminin receptors (Brown et al., 1983; Terranova et al., 1983) on the SC surface, exogenous laminin reaches locally high concentrations sufficient to initiate selfassembly and/or complex formation with other SC-derived basal lamina components; the laminin concentrations used in our experiments were below that required for self-assembly (Yurchenco et al., 1985). It is not known whether the co-deposition of non-triple-helical type IV collagen and HSPG along with laminin was coincidental or was essential for that deposition and the myelination that it enabled. Exogenous type IV collagen may have failed to promote basal lamina formation simply because it did not significantly bind to the SC surface. In addition, type IV collagen secreted by cells and incorporated into developing basal lamina may initially be in a monomeric form, whereas the EHS type IV collagen used in these experiments 
consists predominantly of dimers (Yurchenco and Furthmayr, 1984); it is possible, therefore, that the exogenous type IV collagen was in the wrong form for SCs to utilize for basal lamina assembly. Endogenous, triple-helical type IV collagen, synthesized by the $\mathrm{SC}$ in the presence of ascorbic acid, could initiate basal lamina formation by (1) interacting with laminin and other basal lamina molecules prior to or during secretion, thereby forming complexes (Kleinman et al., 1983, 1986) that could deposit on the SC surface by binding to receptors and/or previously assembled complexes or (2) enabling the efficient transport and secretion of other basal lamina molecules.

The results of Vogel and coworkers (reviewed in Vogel et al., 1987) on the effects of an ascorbic acid-like molecule from embryonic brain on skeletal muscle cell basal lamina formation and differentiation ( $\mathrm{ACh}$ receptor aggregation) show remarkable parallels to our results with SC basal lamina formation and differentiation. Both sets of observations raise the possibility that neurons may control basal lamina production (and thus further differentiation such as myelination) by acting as "purveyors" of ascorbic acid to the cells they contact. Although the neuron could be a source of ascorbic acid for the developing SC in vivo - in the rat, ascorbic acid is synthesized only in the liver (Chatterjee, 1973)-evidence suggests that the neuron does not control whether and when the SC myelinates by simply inducing basal lamina formation. Both myelinating and nonmyelinating (ensheathing) SCs acquire basal laminae (Peters et al., 1976). In addition, SCs in developing peripheral nerve acquire basal lamina at a very early stage, at which time axons abut one another with no intervening SC processes (Cravioto, 1965; Gamble, 1966; Ochoa, 1971), so that SC proliferation, axon sorting, and ensheathment and myelination occur with the SCs always in association with basal lamina (see Martin and Webster, 1973; Webster et al., 1973). Our in vitro experiments, however, have allowed us to study SCs without basal lamina and thus to demonstrate the SC's dependence on this structure for myelination. It may be that basal lamina is required to enable the SC to generate the plasma membrane polarity and cytoskeletal organization needed to execute the complex shape changes that differentiation, including myelin formation, requires (Bunge and Bunge, 1983; Bunge et al., 1986; Eldridge et al., 1987). As we have discussed elsewhere (Bunge and Bunge, 1983), the mutant dystrophic mouse may represent an in vivo corollary of this observation; axons are not myelinated in areas where SCs exhibit basal lamina deficiency. The ability to culture various combinations of normal and dystrophic SCs and neurons and to initiate basal lamina formation by several different means (ascorbic acid, basal lamina matrix, and purified laminin) makes this SC-neuron culture system attractive as a model for the study of mechanisms by which the cell controls, accomplishes, and responds to basal lamina assembly.

\section{References}

Baron-Van Evercooren, A., H. K. Kleinman, H. E. J. Seppa, B. Rentier, and M. Dubois-Dalcq (1982) Fibronectin promotes rat Schwann cell growth and motility. J. Cell Biol. 93: 211-216.

Berg, R. A., M. I. Schwartz, and R. G. Crystal (1980) Regulation of the production of secretory proteins: Intracellular degradation of newly synthesized "defective" collagen. Proc. Natl. Acad. Sci. USA 77: 4746-4750.

Bologna-Sandru, L., H. P. Siegrist, H. Zgraggen, K. Hofmann, U. Wiesmann, D. Dahl, and N. Herschkowitz (1981) Expression of antigenic markers during the development of oligodendrocytes in mouse brain cultures. Brain Res. 210: 217-229.
Bornstein, M. B. (1958) Reconstituted rat-tail collagen used as a substrate for tissue cultures on coverslips. Lab. Invest. 7: 134-137.

Brockes, J. P., M. C. Raff, J. Nichiguchi, and J. Winter (1980) Studies on cultured rat Schwann cells. III. Assays for peripheral myelin proteins. J. Neurocytol. 9: 67-77.

Brockes, J. P., K. J. Fryxell, and G. E. Lemke (1981) Studies on cultured Schwann cells: The induction of myelin synthesis, and the control of their proliferation by a new growth factor. J. Exp. Biol. 95: 215-230.

Brown, S. S., H. L. Malinoff, and M. S. Wicha (1983) Connectin: Cell surface protein that binds both laminin and actin. Proc. Natl. Acad. Sci. USA 80: $5927-5930$.

Brunden, K. R., and J. F. Poduslo (1987) Lysosomal delivery of the major myelin glycoprotein in the absence of myelin assembly: Posttranslational regulation of the level of expression by Schwann cells. J. Cell Biol. 104: 661-669.

Bunge, M. B., A. K. Williams, P. M. Wood, J. Uitto, and J. J. Jeffrey (1980) Comparison of nerve cell and nerve cell plus Schwann cell cultures, with particular emphasis on basal lamina and collagen formation. J. Cell Biol. 84: 184-202.

Bunge, M. B., A. K. Williams, and P. M. Wood (1982) Neuron-Schwann cell interaction in basal lamina formation. Dev. Biol. 92: 449-460.

Bunge, M. B., R. P. Bunge, D. J. Carey, C. J. Cornbrooks, C. F. Eldridge, A. K. Williams, and P. M. Wood (1983) Axonal and nonaxonal influences on Schwann cell development. In Developing and Regenerating Vertebrate Nervous Systems, P. W. Coates, R. R. Markwald, A. D. Kenny, eds., pp. 71-105, Liss, New York.

Bunge, R. P., and M. B. Bunge (1983) Interrelationship between Schwann cell function and extracellular matrix production. Trends Neurosci. 6: 499-505.

Bunge, R. P., and M. B. Bunge (1984) Tissue culture observations relating to pcriphcral nerve development, regeneration and disease. In Peripheral Neuropathy, 2nd ed., P. J. Dyck, P. K. Thomas, E. H. Lambert, R. P. Bunge, eds., Vol. 1, pp. 378-399, Saunders, Philadelphia.

Bunge, R. P., M. B. Bunge, and C. F. Eldridge (1986) Linkage between axonal ensheathment and basal lamina production by Schwann cells. Annu. Rev. Neurosci. 9: 305-328.

Bunge, R. P., C. F. Eldridge, M. D. Ard, and N. Kleitman (1988) Schwann cell contact as a factor in neuronal trophic support and the promotion of neurite growth. In Neurobiology of Amino Acids, Peptides and Trophic Factors, J. Ferrendelli, R. Collins, and E. Johnson, eds., pp. 115-126, Martinus Nijhoff, Boston.

Carey, D. J., and R. P. Bunge (1981) Factors influencing the release of proteins by cultured Schwann cells. J. Cell Biol. 91: 666-672.

Carey, D. J., and M. S. Todd (1987) Schwann cell myelination in a chemically defined medium: Demonstration of a requirement for additives that promote Schwann cell extracellular matrix formation. Dev. Brain Res. 32: 95-102.

Carey, D. J., C. F. Eldridge, C. J. Cornbrooks, R. Timpl, and R. P. Bunge (1983) Biosynthesis of type IV collagen by cultured rat Schwann cells. J. Cell Biol. 97: 473-479.

Carey, D. J., M. S. Todd, and C. M. Rafferty (1986) Schwann cell myelination: Induction by exogenous basement membrane-like extracellular matrix. J. Cell Biol. 102: 2254-2263.

Chatterjee, I. B. (1973) Evolution and the biosynthesis of ascorbic acid. Science 182: 1271-1272.

Cornbrooks, C. J., D. J. Carey, J. A. McDonald, R. Timpl, and R. P. Bunge (1983) In vivo and in vitro observations on laminin production by Schwann cells. Proc. Natl. Acad. Sci. USA 80:3850-3854.

Cravioto, H. (1965) The role of Schwann cells in the development of human peripheral nerves, J. Ultrastruct. Res. 12: 634-651.

Dean, A. C., J. D. Milbrandt, and R. P. Bunge (1986) Axons influence laminin but not collagen IV mRNA levels in Schwann cells. Soc. Neurosci. Abstr. 12: 159.

DeVries, G. H., J. L. Salzer, and R. P. Bunge (1982) Axolemmaenriched fractions isolated from PNS and CNS are mitogenic for Schwann cells. Dev. Brain Res. 3: 295-299.

Ekblom, P., D. Vestueber, and R. Kemler (1986) Cell-matrix interactions and cell adhesion during development. Annu. Rev. Cell Biol. 2: $27-48$.

Eldridge, C. F., and R. P. Bunge (1987) Normal Schwann cells release a factor which stimulates normal Schwann cell proliferation. Anat. Rec. 218: 40a.

Eldridge, C. F., J. R. Sanes, A. Y. Chiu, R. P. Bunge, and C. J. Corn- 
brooks (1986) Basal lamina-associated heparan sulfate proteoglycan in the rat peripheral nervous system: Characterization and localization using monoclonal antibodies. J. Neurocytol. 15: 37-51.

Eldridge, C. F., M. B. Bunge, R. P. Bunge, and P. M. Wood (1987) Differentiation of axon-related Schwann cells in vitro. I. Ascorbic acid regulates basal lamina assembly and myelin formation. J. Cell Biol. 105: 1023-1034.

Eldridge, C. F., R. P. Bunge, and M. B. Bunge (1988) Effects of cis4-hydroxy-L-proline, an inhibitor of Schwann cell differentiation, on the secretion of collagenous and non-collagenous proteins by Schwann cells. Exp. Cell Res. 174: 491-501.

Elsdale, T., and J. Bard (1972) Collagen substrata for studies on cell behavior. J. Cell Biol. 54: 626-637.

Gamble, H. J. (1966) Further electron microscope studies of human foctal peripheral nerves. J. Anat. 100: 487-502.

Hassell, J. R., W. C. Leyshon, S. R. Ledbetter, B. Tyree, S. Suzuki, M. Kato, K. Kimata, and H. K. Kleinman (1985) Isolation of two forms of basement membrane proteoglycans. J. Biol. Chem. 260: 80988105.

Hay, E. D. (1984) Cell-matrix interaction in the embryo: Cell shape, cell surface, cell skeletons, and their role in differentiation. In The Role of Extracellular Matrix in Development, R. L. Trelstead, ed., pp. 1-31, Liss, NY.

Karakashian, M. W., and N. A. Kefalides (1982) Studies on the underhydroxylated basement membrane procollagen synthesized by rat parietal yolk sacs in the presence of $\alpha, \alpha^{\prime}$-dipyridyl. Connect. Tissue Res. 10: 247-262.

Kivirikko, K. I., and R. Myllyla (1982) Post-translational modifications. In Collagen in Health and Disease, J. Wciss and M. Jayson, eds., pp. 101-120, Churchill and Livingstone, New York.

Kleinman, H. K., R. S. Klebe, and G. R. Martin (1981) Role of collagenous matrices in the adhesion and growth of cells. J. Cell Biol. 88: $473-485$.

Kleinman, H. K., M. L. McGarvey, L. A. Liotta, P. G. Robey, K. Tryggvason, and G. R. Martin (1982) Isolation and characterization of type IV procollagen, laminin and heparan sulfate proteoglycan from the EHS sarcoma. Biochemistry 21: 6188-6193.

Kleinman, H. K., M. L. McGarvey, J. R. Hassell, and G. R. Martin (1983) Formation of a supramolecular complex is involved in the reconstitution of basement membrane components. Biochemistry 22 ; $4969-4974$.

Kleinman, H. K., M. L. McGarvey, J. R. Hassell, V. L. Star, F. B. Cannon, G. W. Laurie, and G. R. Martin (1986) Basement membrane complexes with biological activity. Biochemistry 25:312-318.

Ledbetter, S. R., B. Tyree, J. R. Hassell, and E. A. Horigan (1985) Identification of the precursor protein to basement membrane heparan sulfate proteoglycan. J. Biol. Chem. 260: 8106-8113.

Martin, J. R., and H. deF. Webster (1973) Mitotic Schwann cells in developing nerve. Their changes in fine shape (structure) and axon relationships. Dev. Biol. 32: 417-431.

McCarthy, K., and L. Partlow (1976) Neuronal stimulation of $\left[{ }^{3} \mathrm{H}\right]$ thymidine incorporation by primary cultures of highly purified nonneuronal cells. Brain Res. 114: 415-426.

McGarvey, M. L., A. Baron-Van Evercooren, H. K. Kleinman, and M. Dubois-Dalcq (1984) Synthesis and effects of basement membrane components in cultured rat Schwann cells. Dev. Biol. 105: 18-28.

Mirsky, R., J. Winter, E. R. Abney, R. M. Pruss, J. Gavrilovic, and M. C. Raff (1980) Myelin-specific proteins and glycolipids in rat Schwann cells and oligodendrocytes in culture. J. Cell Biol. 84: 483-494.

Moya, F., M. B. Bunge, and R. P. Bunge (1980) Schwann cells proliferate but fail to differentiate in defined medium. Proc. Natl. Acad. Sci. USA 77: 6902-6906.

Neblock, D. S., and R. A. Berg (1982) The effect of cis-4-hydroxy-Lproline on intracellular degradation of newly synthesized collagen by freshly isolated chick tendon fibroblasts. Connect. Tissue Res. 10: 297-301.

Ochoa, J. (1971) The sural nerve of the human fetus: Electron microscope observations and counts of axons. J. Anat. 108: 231-245.

Paul, J. (1970) Cell and Tissue Culture, Williams \& Wilkins, Baltimore.

Peters, A., S. L. Palay and H. deF. Webster (1976) The Fine Structure of the Nervous System: The Neurons and Supporting Cells, Saunders, Philadelphia.
Poduslo, J. F., P. J. Dyck, and C. T. Berg (1985) Regulation of myelination: Schwann cell transition from a myelin-maintaining state to a quiescent state after permanent nerve transection. J. Neurochem. 44: $388-400$.

Politis, M. J., N. Sternberger, K. Ederle, and P. S. Spencer (1982) Studies on the control of myelinogenesis. IV. Neuronal induction of Schwann cell myelin-specific protein synthesis during nerve fiber regeneration. J. Neurosci. 2: 1252-1266.

Prockop, D. J., R. A. Berg, K. I. Kivirikko, and J. Uitto (1976) Intracellular steps in the biosynthesis of collagen. In Biochemistry of Collagen. G. N. Ramachandran and A. H. Reddi, eds. pp. 163-273, Plenum, New York.

Ratner, N., A. Elbein, M. B. Bunge, S. Porter, R. P. Bunge, and L. Glaser (1986) Specific asparagine-linked oligosaccharides are not required for certain neuron-neuron and neuron-Schwann cell interactions. J. Cell Biol. 103: 159-170.

Ratner, N., C. Eldridge, R. P. Bunge, and L. Glaser (1987) Effects of an inhibitor of proteoglycan biosynthesis on neuron-induced Schwann cell proliferation and basal lamina formation by Schwann cells. In Mesenchymal/Epithelial Interactions in Neural Development, J. R. Wolff and J. Sievers, eds., pp. 127-139, Springer-Verlag, Berlin.

Salzer, J. L., and R. P. Bunge (1980) Studies of Schwann cell proliferation: I. An analysis in tissue culture of proliferation during development, Wallerian degeneration, and direct injury. J. Cell Biol. 84: 739-752.

Salzer, J. L., R. P. Bunge, and L. Glaser (1980a) Studies of Schwann cell proliferation: III. Evidence for the surface localization of the neurite mitogen. J. Cell Biol. 84: 767-778.

Salzer, J. L., A. K. Williams, L. Glaser, and R. P. Bunge (1980b) Studies of Schwann cell proliferation: II. Characterization of the stimulation and specificity of the response to a neurite membrane fraction. J. Cell Biol. 84: 753-766.

Sanes, J. R. (1983) Roles of extracellular matrix in neural development. Annu. Rev. Physiol. 45: 581-600.

Schlaepfer, W. W. (1983) Neurofilaments and the axonal cytoskeleton as determinants of stability and growth in regenerating axons. In Spinal Cord Reconstruction, C. C. Kao, R. P. Bunge, and P. J. Reier, eds., pp. 59-73, Raven, New York.

Terranova, V. P., C. N. Rao, T. Kalebic, I. M. Margulies, and L. A. Liotta (1983) Laminin receptor on human breast carcinoma cells. Proc. Natl. Acad. Sci. USA 80: 444-448.

Timpl, R., R. Heilwig, P. G. Robey, S. F. Rennard, J. M. Foidart, and G. M. Martin (1979) Laminin-A glycoprotein from basement membranes. J. Biol. Chem. 254: 9933-9937.

Tsilibary, E. C., and A. S. Charonis (1986) The role of the main noncollagenous domain $(\mathrm{NCl})$ in type IV collagen self-assembly. $\mathrm{J}$. Cell Biol. 103: 2467-2473.

Vogel, Z., M. P. Daniels, T. Chen, Z.-Y. Xi, E. Bachar, L. Ben-David, N. Rosenberg, M. Krause, D. Duksin, and C. Kalcheim (1987) Ascorbate-like factor from embryonic brain. Role in collagen formation, basement membrane deposition, and acetylcholine receptor aggregation by muscle cells. Ann. NY Acad. Sci. 498: 13-27.

Webster, H. deF., and J. T. Favilla (1984) Development of peripheral nerve fibers. In Peripheral Neuropathy, 2nd ed., P. J. Dyck, P. K. Thomas, E. H. Lambert, and R. P. Bunge, eds., Vol. 1, pp. 329-359, Saunders, Philadelphia.

Webster, H. deF., J. R. Martin, and M. F. O'Connell (1973) The relationships between interphase Schwann cells and axons before myelination: A quantitative electron microscopic study. Dev. Biol. 32: $401-416$.

Wood, P. M., and R. P. Bunge (1975) Evidence that sensory axons are mitogenic for Schwann cells. Nature 256: 662-664.

Wood, P. M., and A. K. Williams (1984) Oligodendrocyte proliferation and CNS myelination in cultures containing dissociated embryonic neuroglia and dorsal root ganglion neurons. Dev. Brain Res. 12:225241.

Yurchenco, P. D., and H. Furthmayr (1984) Self-assembly of basement membrane collagen. Biochemistry 23: 1839-1850.

Yurchenco, P. D., E. C. Tsilibary, A. S. Charonis, and H. Furthmayr (1985) Laminin polymerization in vitro. J. Biol. Chem. 260: 76367644. 